\title{
Does transparent hidden matter generate optical scintillation?
}

\author{
M. Moniez ${ }^{\star}$ \\ Laboratoire de l'Accélérateur Linéaire, IN2P3-CNRS, Université de Paris-Sud, BP 34, 91898 Orsay Cedex, France
}

Received 17 February 2003 / Accepted 4 September 2003

\begin{abstract}
Stars twinkle because their light goes through the atmosphere. The same phenomenon is expected when the light of extra-galactic stars goes through a Galactic - disk or halo - refractive medium. Because of the large distances involved here, the length and time scales of the optical intensity fluctuations resulting from the wave distortions are accessible to current technology. In this paper, we discuss the different possible scintillation regimes and we focus on the so-called strong diffractive regime that is likely to produce large intensity contrasts. The critical relationship between the source angular size and the intensity contrast in optical wavelengths is also discussed in detail. We propose to monitor small extra-galactic stars every $\sim 10 \mathrm{~s}$ to search for intensity scintillation produced by molecular hydrogen clouds. We discuss means to discriminate such hidden matter signals from the foreground effects on light propagation. Appropriate observation of the scintillation process described here should allow one to detect column density stochastic variations in Galactic molecular clouds of the order of $\sim 3 \times 10^{-5} \mathrm{~g} / \mathrm{cm}^{2}$, that is $10^{19}$ molecules $/ \mathrm{cm}^{2}$ per $\sim 10000 \mathrm{~km}$ transverse distance.
\end{abstract}

Key words. cosmology: dark matter - Galaxy: disk - Galaxy: halo - ISM: clouds - ISM: molecules

\section{Introduction: The Galactic hidden matter problem and the baryons}

The study of rotation curves of spiral galaxies has led to the hypothesis of massive extended halos of "dark matter" (e.g. Primack et al. 1988). From the big-bang nucleosynthesis theory and the measured primordial abundances of the light elements, it is also established that baryons in the Universe are at least ten times more abundant than in the visible matter (stars, dust and gas) (Olive 2000). This deficit of baryonic matter in the Universe is approximately equal to the deficit of gravitational matter in our Galaxy. This coincidence was one of the major motivations for the first microlensing searches for baryonic hidden matter under the form of Massive Compact Halo Objects (MACHOs). Considering the results of these searches (Lasserre et al. 2000; Afonso et al. 2003; Alcock et al. 2000), it seems that the only constituent that could contribute quite significantly to the Galactic baryonic hidden matter is the cool molecular hydrogen $\left(\mathrm{H}_{2}\right)$. Indeed, this material is very difficult to detect, due to the symmetry of the $\mathrm{H}_{2}$ molecule that cancels the dipolar electric transitions, the only ones to be excited in a cold medium $(\sim 10 \mathrm{~K})$. The perspectives for detecting cold $\mathrm{H}_{2}$ in outer Galactic disks have been reviewed by Combes \& Pfenniger (1997). Most of the techniques currently used to estimate $\mathrm{H}_{2}$ Galactic contribution are indirect detections using tracers such as $\mathrm{CO}$ molecule or dust, that imply specific hypotheses. A possible direct method using the ultra-fine transitions of the nuclear spins (from parallel to anti-parallel) concerns radio emission or absorption $(\lambda=0.5 \mathrm{~km}$ and $5.5 \mathrm{~km})$

\footnotetext{
* e-mail: moniez@lal.in2p3.fr
}

that is impossible to detect in the terrestrial environment. Detection of Galactic $\mathrm{H}_{2}$ clouds in front of quasars is not easily feasible, because the absorption lines have wavelengths shorter than $110 \mathrm{~nm}$. In summary, the hypothesis of a leading contribution to the halo mass due to cold molecular hydrogen is not yet ruled out by any of the currently used methods. A hierarchical structure for cold molecular hydrogen has been suggested by Pfenniger \& Combes (1994) to fill the Galactic thick disk and De Paolis et al. (1995 and 1998) have considered cold molecular hydrogen clouds as possible candidates for the Galactic halo dark matter. According to Pfenniger \& Combes (1994) model, the gas could form "clumpuscules" of 10 AU size at the smallest scale, with a column density of $10^{24-25} \mathrm{~cm}^{-2}$, and a surface filling factor less than $1 \%$. We propose to search for such cool molecular clouds in the thick disk and in the halo through their diffraction and refraction effects on the propagation of remote stars light. The method that will be discussed in this paper is of more general use and could be used to detect other types of transparent structures. Nevertheless, our immediate aim is to demonstrate its feasibility and its sensitivity to gaseous structures that are considered as viable candidates for the Galactic hidden matter.

\section{Detection mode of extended $\mathrm{H}_{2}$ clouds: Principle}

Due to index refraction effects, an inhomogeneous transparent (gaseous) medium distorts the wave-fronts of incident electro-magnetic waves. When observing a remote source located behind a gaseous structure (hereafter called screen), 
the luminous amplitude results from the propagation of the distorted wave-front. Diffraction theory predicts that interference patterns should form and also possibly longer scale refraction effects (prism and lens-like). In the following, we will study the visibility of the wave-front distortions through the intensity contrasts produced on Earth. We will particularly discuss the critical aspect of the spatial coherence and show that only the light of faint stars (remote and small) can provide a detectable signal of intensity variations due to a refractive structure.

Before developing the scintillation mechanisms, we stress that the effect of a refraction process is cumulative, in the sense that the more material the light encounters, the larger are the wave distortions. If there is no dust, the sources behind a gaseous structure are always visible provided that the wavelength is not resonant with a transition process of the medium constituents.

\section{Refraction by Galactic hidden gas}

The elementary process responsible for the refraction index effect is the polarizability of molecules. After propagation along a distance $l$, the optical path difference between the vacuum and a medium characterized by a number density $N$ (number of molecules per volume unit) and polarizability $\alpha$ is

$\delta=2 \pi N l \alpha$.

It depends only on the total column number density $N l$ of molecules and on their average polarizability $\alpha$, but not on the details of the density distribution along the propagation path. In the present case, the density is so low $\left(\sim 10^{9}\right.$ molecules $\left./ \mathrm{cm}^{3}\right)$ that the medium cannot be considered as continuous at the optical wavelength scale (the average distance between molecules is $\sim 10 \mu \gg \lambda$ ). Then it is not possible to consider that the polarizability in a small region (compared with the optical wavelength) results from the average of polarizabilities over the molecule orientations as it is usually considered in dense mediums $^{1}$. Nevertheless, we study the result of the diffusion at extremely large distances from the screen and precisely towards the initial propagation direction, where the diffusion is coherent; then the collective effect of successive diffusions by randomly oriented molecules scattered along the propagating path is the same than the diffusion by molecules with the average polarizability (Hamilton 1960).

For a gas only made of $\mathrm{H}_{2}$ molecules, $\alpha=0.802 \times 10^{-24} \mathrm{~cm}^{3}$ (CRC Handbook 1998-99) ${ }^{2}$.

If the Galactic halo is as described by Caldwell \& Coulson (1986) (the so-called standard spherical halo) and if it is completely made of $\mathrm{H}_{2}$, then the average column density from the Sun to LMC (respectively SMC and M31) is $0.0253 \mathrm{~g} / \mathrm{cm}^{2}$ (resp. 0.0307 and 0.0206), corresponding to $N l=7.62 \times$ $10^{21}$ molecules per $\mathrm{cm}^{2}$ (resp. $9.25 \times 10^{21}$ and $6.20 \times 10^{21}$ ) or

\footnotetext{
${ }_{1}$ As the $\mathrm{H}_{2}$ molecule is not spherical, its polarization may not be aligned with the electric field, and the polarization averaged over the molecule orientations is different than the individual polarizations.

${ }^{2}$ For a $\mathrm{H}_{2} / \mathrm{He}$ mixing with $24 \% \mathrm{He}$ by mass - corresponding to the primordial abundances $-\langle\alpha\rangle=0.720 \times 10^{-24} \mathrm{~cm}^{3}$, and subsequent calculations should be renormalized accordingly.
}

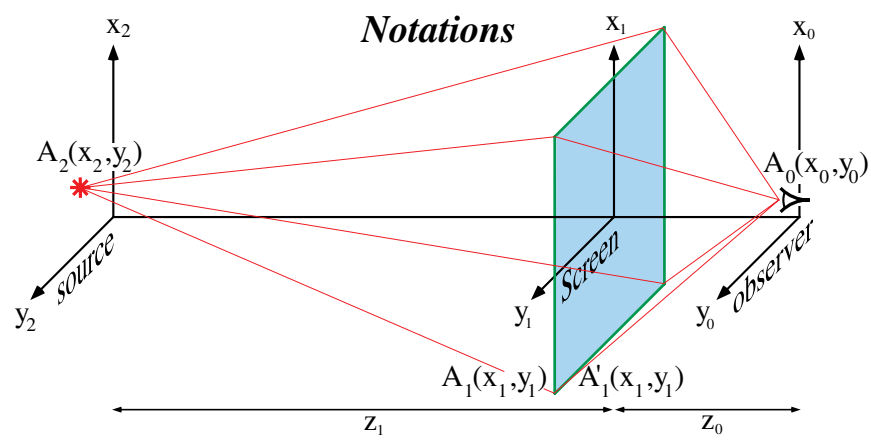

Fig. 1. Notations: The source is located in the $\left(x_{2}, y_{2}\right)$ plane, the screen contains the diffusive structure, and the observer is located in the $\left(x_{0}, y_{0}\right)$ plane. $A_{1}\left(x_{1}, y_{1}\right)$ and $A_{1}^{\prime}\left(x_{1}, y_{1}\right)$ are the amplitudes before and after screen crossing.

to a column of $2.83 \mathrm{~m}$ (resp. $3.44 \mathrm{~m}$ and $2.31 \mathrm{~m}$ ) of $\mathrm{H}_{2}$ under normal pressure and temperature conditions. The extra optical path induced by this medium with respect to the vacuum is $\delta \simeq 0.4 \mathrm{~mm} \simeq 768 \lambda$ at $\lambda=500 \mathrm{~nm}$ towards LMC (resp. $932 \lambda$ and $625 \lambda$ ). The same orders of magnitude are expected if hidden matter lies in the thick disk instead of the halo.

These values are average values and we have to take into account the structuration of this gas. In the Pfenniger-Combes model, the smallest $\mathrm{H}_{2}$ structures are $10 \mathrm{AU}$ wide and have a Jupiter mass. Therefore their surface filling factor is less than $1 \%$. This means that we expect concentration factors of the column density of 100 at least $\left(\Rightarrow N l \sim 10^{24} \mathrm{~cm}^{-2}\right)$ for $1 \%$ of the sky fields. For such a structure the average transverse gradient of optical path differences is of order of

$$
\frac{800 \times \lambda \times 100 \text { (concentration) }}{5 \mathrm{AU}} \sim 1 \lambda \text { per } 10000 \mathrm{~km},
$$

for $\lambda=500 \mathrm{~nm}$. As we will see in the next section, this fits with the typical optical path fluctuations that can produce interference patterns for a $\mathrm{H}_{2}$ cloud located in the Galactic disks or Galactic halo.

\section{Diffractive scintillation: Fresnel diffraction applied to a stellar source and a Galactic screen}

\subsection{General formalism, notations}

Let $A_{2}\left(x_{2}, y_{2}\right)$ be the luminous (complex) amplitude produced in the source plane (see Fig. 1). For a monochromatic (wavelength $\lambda)$ point-like source of amplitude $A_{2}$ located at $\left(x_{2}, y_{2}\right)$ in the source plane, omitting the time periodic factor $\mathrm{e}^{-i \omega t}$, the amplitude on the screen before diffusion is given by the spherical wave equation:

$A_{1}\left(x_{1}, y_{1}\right)=A_{2} \mathrm{e}^{i k r_{12}} / r_{12} \simeq A_{2} \mathrm{e}^{i k r_{12}} / z_{1}$,

where $\boldsymbol{k}$ is the wave vector $(k=2 \pi / \lambda)$, and

$$
\begin{aligned}
r_{12} & =\sqrt{z_{1}^{2}+\left(x_{1}-x_{2}\right)^{2}+\left(y_{1}-y_{2}\right)^{2}} \\
& =z_{1} \sqrt{1+\left(\frac{x_{1}-x_{2}}{z_{1}}\right)^{2}+\left(\frac{y_{1}-y_{2}}{z_{1}}\right)^{2}}
\end{aligned}
$$


can be approximated by $z_{1}$ in the denominator because we will always be in the situation where $z_{1} \gg x_{1}$ and $y_{1}$. The effect of the screen on the wave propagation can be represented by a phase delay that depends only on $x_{1}$ and $y_{1}$ :

$A_{1}^{\prime}\left(x_{1}, y_{1}\right)=\frac{A_{2} \mathrm{e}^{i k r_{12}}}{z_{1}} \mathrm{e}^{i k \delta\left(x_{1}, y_{1}\right)}$,

where $\delta\left(x_{1}, y_{1}\right)$ is the extra optical path due to the screen. The amplitude after subsequent propagation in vacuum is given by the Huygens-Fresnel diffraction principle:

$A_{0}\left(x_{0}, y_{0}\right)=\int_{-\infty}^{+\infty} \int_{-\infty}^{+\infty} A_{1}^{\prime}\left(x_{1}, y_{1}\right) \frac{\mathrm{e}^{i k r_{01}}}{i \lambda z_{0}} \mathrm{~d} x_{1} \mathrm{~d} y_{1}$,

where

$r_{01}=z_{0} \sqrt{1+\left(\frac{x_{0}-x_{1}}{z_{0}}\right)^{2}+\left(\frac{y_{0}-y_{1}}{z_{0}}\right)^{2}}$.

\subsection{Approximations}

The stationary phase approximation states that the main contribution to integral (5) comes from the $\left(x_{1}, y_{1}\right)$ domain where the phase term of the integrand does not oscillate too fast. In this domain, the Fresnel approximation is valid. It consists in keeping only the first order development of the square root in the expression of $r_{01}$ :

$r_{01} \simeq z_{0}\left[1+\frac{1}{2}\left(\frac{x_{0}-x_{1}}{z_{0}}\right)^{2}+\frac{1}{2}\left(\frac{y_{0}-y_{1}}{z_{0}}\right)^{2}\right]$.

This approximation is in principle only valid if the next order terms are negligible. But as the integrand oscillates very fast as soon as $\left(x_{0}-x_{1}\right)^{2}+\left(y_{0}-y_{1}\right)^{2}>\lambda z_{0}$, the contribution of the integral vanishes before the Fresnel approximation fails. This is why we can keep the infinite limits of the integration domain, without the "artificial" need of a pupil ${ }^{3}$. Within the framework of Fresnel approximation, the amplitude in the observer's plane is

$$
\begin{aligned}
A_{0}\left(x_{0}, y_{0}\right) & =\frac{\mathrm{e}^{i k z_{0}}}{i \lambda z_{0}} \iint_{-\infty}^{+\infty} A_{1}^{\prime}\left(x_{1}, y_{1}\right) \mathrm{e}^{\frac{i k}{2 z_{0}}\left[\left(x_{0}-x_{1}\right)^{2}+\left(y_{0}-y_{1}\right)^{2}\right]} \mathrm{d} x_{1} \mathrm{~d} y_{1} \\
& =\frac{\mathrm{e}^{i k z_{0}}}{2 i \pi R_{\mathrm{F}}^{2}} \iint_{-\infty}^{+\infty} A_{1}^{\prime}\left(x_{1}, y_{1}\right) \mathrm{e}^{i \frac{\left(x_{0}-x_{1}\right)^{2}+\left(y_{0}-y_{1}\right)^{2}}{2 R_{\mathrm{F}}^{2}}} \mathrm{~d} x_{1} \mathrm{~d} y_{1},
\end{aligned}
$$

where $R_{\mathrm{F}}=\sqrt{z_{0} / k}=\sqrt{\lambda z_{0} / 2 \pi}$ is the Fresnel radius. $R_{\mathrm{F}}$ is of order of $1500 \mathrm{~km}$ to $15000 \mathrm{~km}$ at $\lambda=500 \mathrm{~nm}$, for a screen located between $1 \mathrm{kpc}$ to $100 \mathrm{kpc}$. This length scale characterizes the $\left(x_{1}, y_{1}\right)$ domain that contributes to the integral (a few Fresnel radii). Then it is clear that the Fraunhofer approximation - that assumes the contribution of $\left(x_{1}^{2}+y_{1}^{2}\right) / 2 R_{\mathrm{F}}^{2}$ to the phase term development as a constant over the pupil - is not valid here. In return, as we will consider sources located much further from the observer than the screen, we can approximate the spherical incident wave to a plane wave. For a point-like source located at $\left(x_{2}, y_{2}\right)=(0,0)$, we therefore assume that $A_{1}\left(x_{1}, y_{1}\right)=A$

\footnotetext{
3 This property has been checked in the simple example considered below.
}

is a real constant just before the screen. The amplitude in the observation plane is then

$A_{0}\left(x_{0}, y_{0}\right)=\frac{A \mathrm{e}^{i k z_{0}}}{2 i \pi R_{\mathrm{F}}^{2}} \iint_{-\infty}^{+\infty} \mathrm{e}^{i k \delta\left(x_{1}, y_{1}\right)} \mathrm{e}^{i \frac{\left(x_{0}-x_{1}\right)^{2}+\left(y_{0}-y_{1}\right)^{2}}{2 R_{\mathrm{F}}^{2}}} \mathrm{~d} x_{1} \mathrm{~d} y_{1}$.

The resulting amplitude is affected by strong interferences in the observer's plane (the so-called speckle) if $\delta\left(x_{1}, y_{1}\right)$ varies stochastically of order of $\lambda$ within the Fresnel domain. This is precisely the same order of magnitude as the average gradient that characterizes the $\mathrm{cool} \mathrm{H}_{2}$ structures.

The above expression corresponds to a point-like monochromatic source. We will now consider a simple configuration to discuss spatial and time coherence effects that severely limit the visibility of diffraction fringes.

\section{Diffractive scintillation: A simple, pedagogical (and realistic) screen example}

Fresnel formalism cannot provide a simple procedure to take into account the source size. In this section, we analyze the effect of the source size in the case of a simple phase screen. Let us assume that the screen is a step of optical path $\delta$ parallel to the $y_{1}$ axis, described by a Heaviside distribution in the $\left(x_{1}, y_{1}\right)$ plane $\delta\left(x_{1}, y_{1}\right)=\delta \times H\left(x_{1}\right)$. This case is realistic since, at the Fresnel scale, the edge of a gaseous structure can be considered as a straight line that divides the plane into two regions. The step approaches the effect of a "strong" local gradient of the optical path. The case of a ramp instead of a step has also been examined, but we will only show here an example of diffraction pattern, because the calculation of the source size effect is more complicated in this case. A more complete discussion is proposed below, that concerns a wide domain of screen models with stochastic optical path variations. Nevertheless, this example will be our guide for feasibility studies.

\subsection{Point-like, monochromatic source}

The integral (9) that corresponds to a monochromatic pointsource can easily be separated into a product of two integrals. The integral along $y$ can be estimated by noticing that in absence of screen there should be no effect on the propagation. We thus get the following relation

$\left[\int_{-\infty}^{+\infty} \mathrm{e}^{i \frac{\left(x_{0}-x_{1}\right)^{2}}{2 R_{\mathrm{F}}^{2}}} \mathrm{~d} x_{1}\right]^{2}=2 i \pi R_{\mathrm{F}}^{2}$

Splitting the integral along $x$ into two parts (for $x_{1}<0$ and $x_{1}>0$ ) we get the expression

$A_{0}\left(x_{0}, y_{0}\right)=A \mathrm{e}^{i k z_{0}}+\frac{A \mathrm{e}^{i k z_{0}}}{\sqrt{2 i \pi} R_{\mathrm{F}}} \int_{0}^{+\infty}\left[\mathrm{e}^{i k \delta}-1\right] \mathrm{e}^{i \frac{\left(x_{0}-x_{1}\right)^{2}}{2 R_{\mathrm{F}}^{2}}} \mathrm{~d} x_{1}$.

It follows that the amplitude in the observer's plane can be expressed by:

$A_{0}\left(x_{0}, y_{0}\right)=A \mathrm{e}^{i k z_{0}}$

$\times\left[1+\frac{\mathrm{e}^{i k \delta}-1}{2}\left[1+S\left(X_{0}\right)+C\left(X_{0}\right)-i\left(S\left(X_{0}\right)-C\left(X_{0}\right)\right)\right]\right]$, 
where $X_{0}$ is the reduced variable, defined by

$X_{0}=x_{0} /\left(\sqrt{\pi} R_{\mathrm{F}}\right)$,

$S$ and $C$ are the Fresnel integrals

$S(X)=\int_{0}^{X} \sin \frac{\pi t^{2}}{2} \mathrm{~d} t$,

$C(X)=\int_{0}^{X} \cos \frac{\pi t^{2}}{2} \mathrm{~d} t$

The intensity in the observer's plane is

$I_{0}\left(x_{0}, y_{0}\right)=A_{0}\left(x_{0}, y_{0}\right) \times A_{0}^{*}\left(x_{0}, y_{0}\right)=A^{2} \times i_{0}\left(X_{0}\right)$,

where

$$
\begin{aligned}
i_{0}\left(X_{0}\right)= & 1-\left(S\left(X_{0}\right)-C\left(X_{0}\right)\right) \sin (k \delta) \\
& +\left[S\left(X_{0}\right)^{2}+C\left(X_{0}\right)^{2}-\frac{1}{2}\right][1-\cos (k \delta)] .
\end{aligned}
$$

Figure 2 displays the variations of this intensity in the observer's plane for $\delta=\lambda / 4$, and shows the contrast of the diffraction pattern as a function of the step size $\delta$. The inter-fringe is -in a natural way- close to the length scale defined by $\sqrt{\pi} R_{\mathrm{F}}$. Figure 3 also shows the diffraction pattern produced by a prism whose edge is the $y_{1}$ axis, with an optical path variation $\delta=\lambda / 2$ per one unit of $\sqrt{\pi} R_{\mathrm{F}}$. Such a gradient is comparable with the average gradient expected from the width variation of $10 \mathrm{AU}$ gas structures in the Galactic thick disk. One should notice here that diffraction patterns take place if the second derivative of the optical path is different from zero. Discontinuity (of the optical path or of its derivatives, as in our examples) is not necessary to get such patterns.

\subsection{Disc-source. Spatial coherence}

A simple geometrical construction suggests that the diffraction pattern produced in the observer's plane by a point source located at $\left(x_{2}, y_{2}\right)$ is the same as the one produced by a point source at the origin, but translated by $\left(-x_{2} z_{0} / z_{1},-y_{2} z_{0} / z_{1}\right)$. Actually, it is easy to check that, within the Fresnel approximation - using expressions (3), (6), (7) -, the difference between the phase $k r_{01}+k r_{12}+\delta\left(x_{1}, y_{1}\right)$ along the optical path defined by $\left(x_{2}, y_{2}\right),\left(x_{1}, y_{1}\right)$ and $\left(x_{0}, y_{0}\right)$ and the phase along the path defined by $(0,0),\left(x_{1}, y_{1}\right)$ and $\left(x_{0}+x_{2} z_{0} / z_{1}, y_{0}+y_{2} z_{0} / z_{1}\right)$ is independent of the point $\left(x_{1}, y_{1}\right)$. This constant phase difference term can be factorized in integral (8), and it follows that it is the only difference between the amplitude diffracted at $\left(x_{0}, y_{0}\right)$ from a source located at $\left(x_{2}, y_{2}\right)$ and the one diffracted at $\left(x_{0}+\right.$ $\left.x_{2} z_{0} / z_{1}, y_{0}+y_{2} z_{0} / z_{1}\right)$ from a source at the origin. The intensity of the diffracted wave from a point-source located at $\left(x_{2}, y_{2}\right)$ is then given by

$I_{0}\left(x_{0}, y_{0}\right)\left[\right.$ source at $\left.\left(x_{2}, y_{2}\right)\right]$

$=I_{0}\left(x_{0}+x_{2} \frac{z_{0}}{z_{1}}, y_{0}+x_{2} \frac{z_{0}}{z_{1}}\right)$ [source at $\left.(0,0)\right]$.

An astrophysical source can (almost always) be considered as a uniform disk (radius $r_{\mathrm{S}}$ ) of incoherent point-source elements.
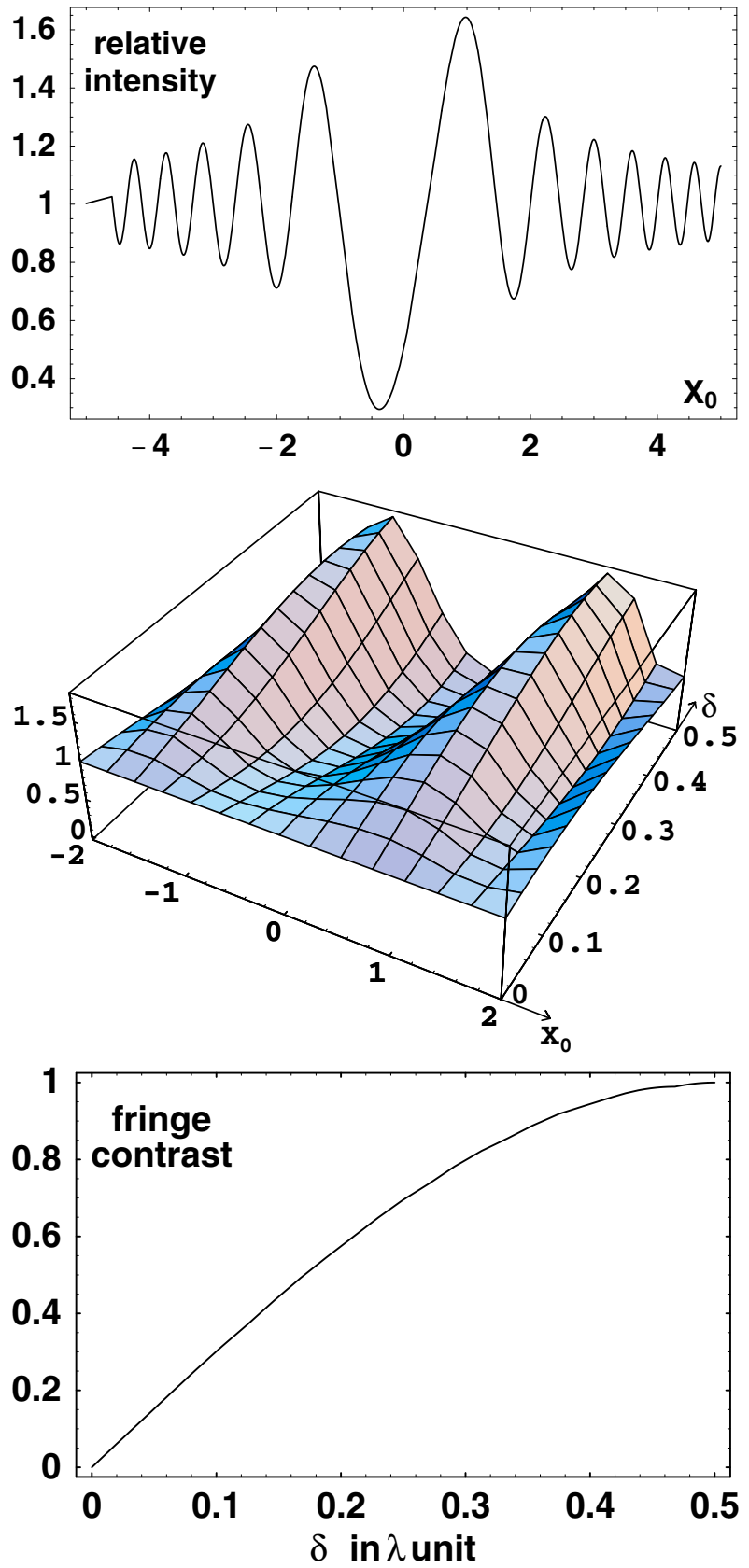

Fig. 2. Top: Diffraction pattern produced in the observer's plane, perpendicularly to a step of optical path $\delta=\lambda / 4$. The $X_{0}-$ axis origin is the intercept of the source-step line with the observer's plane. $X_{0}=1$ corresponds to $x_{0}=\sqrt{\pi} R_{\mathrm{F}}$. Middle: Diffraction pattern as a function of the step size $\delta$ (in depth, from 0 to 0.5 in $\lambda$ units). Bottom: Contrast of the diffraction pattern as a function of the step size $\delta$.

We then have to integrate the intensities from the source elements to get the resulting diffraction pattern. As elements aligned parallel to the $y$-axis give the same diffraction pattern, the total intensity is given by

$\mathcal{I}\left(x_{0}, y_{0}\right)=\frac{I}{\pi r_{\mathrm{S}}^{2}} \int_{-r_{\mathrm{S}}}^{r_{\mathrm{S}}} 2 \sqrt{r_{\mathrm{S}}^{2}-x_{2}^{2}} \times i_{0}\left(\frac{x_{0}+x_{2} \frac{z_{0}}{z_{1}}}{\sqrt{\pi} R_{\mathrm{F}}}\right) \mathrm{d} x_{2}$ 
Table 1. Examples of sources at different distances. - For LMC/SMC and M 31 we have chosen two series of sources with comparable apparent luminosities $\left(M_{V} \sim 18.5\right.$ and $\left.\sim 20.5\right)$. - The size of a SNIa envelope is estimated assuming $10000 \mathrm{~km} \mathrm{~s}^{-1}$ expansion rate during 20 days (Filippenko 1997). - The angular and luminosity distances of the Einstein cross multi-image quasar are estimated with the cosmological parameters of Bennett et al. (2003). The upper size of the continuum source is constrained by microlensing studies. The absolute magnitude given here is only indicative, as it does not take into account the magnification due to gravitational lensing.

\begin{tabular}{|c|c|c|c|c|c|c|c|c|}
\hline \multirow[b]{2}{*}{ Source } & \multirow[b]{2}{*}{ Distance } & \multirow{2}{*}{$\begin{array}{l}\text { Distance } \\
\text { modulus }\end{array}$} & \multirow{2}{*}{$\begin{array}{l}\text { Source or } \\
\text { stellar type }\end{array}$} & \multirow{2}{*}{$\begin{array}{c}\text { Absolute } \\
\operatorname{mag} V\end{array}$} & \multirow{2}{*}{$\begin{array}{l}\text { Apparent } \\
\operatorname{mag} V\end{array}$} & \multirow[b]{2}{*}{ Size } & \multicolumn{2}{|c|}{ Angular size $\theta_{\mathrm{S}}$} \\
\hline & & & & & & & $\mathrm{rad}$ & " \\
\hline Nearby star & $10 \mathrm{pc}$ & 0 & M5V & 12.3 & 12.3 & $0.27 r_{\odot}$ & $6.1 \times 10^{-10}$ & $1.3 \times 10^{-4}$ \\
\hline Galactic star & $8 \mathrm{kpc}$ & 14.5 & K0V & 5.9 & 20.4 & $0.85 r_{\odot}$ & $2.4 \times 10^{-12}$ & $5.0 \times 10^{-7}$ \\
\hline \multirow[t]{2}{*}{ LMC star } & \multirow[t]{2}{*}{$55 \mathrm{kpc}$} & \multirow[t]{2}{*}{18.7} & B8V & -0.25 & 18.45 & $3.0 r_{\odot}$ & $1.2 \times 10^{-12}$ & $2.5 \times 10^{-7}$ \\
\hline & & & $\mathrm{A} 5 \mathrm{~V}$ & 1.95 & 20.65 & $1.7 r_{\odot}$ & $7 \times 10^{-13}$ & $1.4 \times 10^{-7}$ \\
\hline \multirow[t]{2}{*}{ SMC star } & \multirow[t]{2}{*}{$58 \mathrm{kpc}$} & \multirow[t]{2}{*}{18.8} & $\mathrm{~B} 8 \mathrm{~V}$ & -0.25 & 18.55 & $3.0 r_{\odot}$ & $1.1 \times 10^{-12}$ & $2.3 \times 10^{-7}$ \\
\hline & & & A5V & 1.95 & 20.75 & $1.7 r_{\odot}$ & $7 \times 10^{-13}$ & $1.4 \times 10^{-7}$ \\
\hline \multirow[t]{2}{*}{ M31 star } & \multirow[t]{2}{*}{$725 \mathrm{kpc}$} & \multirow[t]{2}{*}{24.3} & $\mathrm{O} 5 \mathrm{~V}$ & -5.7 & 18.6 & 12. $r_{\odot}$ & $3.7 \times 10^{-13}$ & $7.6 \times 10^{-8}$ \\
\hline & & & B0V & -4.0 & 20.3 & $7.4 r_{\odot}$ & $2.3 \times 10^{-13}$ & $4.7 \times 10^{-8}$ \\
\hline $\begin{array}{c}\text { SNIa@max } \\
\text { at } z=0.2\end{array}$ & $0.9 \mathrm{Gpc}$ & 39.8 & SNIa & -19.2 & 20.6 & $\sim 2 \times 10^{10} \mathrm{~km}$ & $7.2 \times 10^{-13}$ & $1.5 \times 10^{-7}$ \\
\hline $\begin{array}{c}\text { Einstein cross A } \\
\quad z=1.695\end{array}$ & $1.75 \mathrm{Gpc}$ & 45.5 & quasar & $\sim-28$. & $\sim 17$ & $\begin{array}{l}<6 \times 10^{10} \mathrm{~km}^{a} \\
<3 \times 10^{11} \mathrm{~km}^{b}\end{array}$ & $\begin{array}{l}<1.1 \times 10^{-12} \\
<5.6 \times 10^{-12}\end{array}$ & $\begin{array}{l}<2.3 \times 10^{-7} \\
<1.1 \times 10^{-6}\end{array}$ \\
\hline
\end{tabular}

${ }^{a}$ Wyithe et al. (2000).

${ }^{b}$ Atsunori (2001).

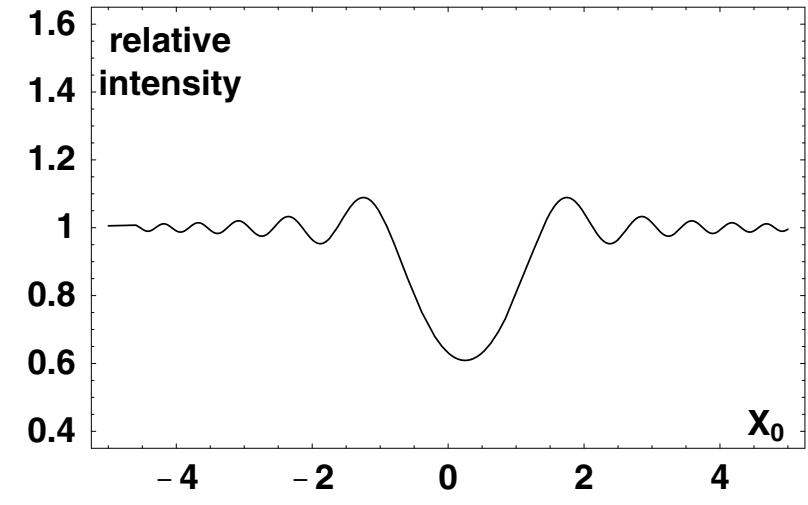

Fig. 3. Diffraction pattern produced perpendicularly to a prism of optical path with $\delta=X_{0} \times \lambda / 2$ for $X_{0}>0$. The $X_{0}$-axis origin is the intercept of the source-corner line with the observer's plane. $X_{0}=1$ corresponds to $x_{0}=\sqrt{\pi} R_{\mathrm{F}}$.

where $I$ is the total intensity emitted by the object on the screen and $i_{0}$ is given by expression (17). Using the reduced variables $X_{0}=\frac{x_{0}}{\sqrt{\pi} R_{\mathrm{F}}}$ and $X_{2}=\frac{x_{2}}{\sqrt{\pi} R_{\mathrm{F}}} \times \frac{z_{0}}{z_{1}}$

leads to

$\mathcal{I}\left(x_{0}, y_{0}\right)=\frac{2 I}{\pi R_{\mathrm{S}}} \int_{-R_{\mathrm{S}}}^{R_{\mathrm{S}}} \sqrt{1-\left[\frac{X_{2}}{R_{\mathrm{S}}}\right]^{2}} i_{0}\left(X_{0}+X_{2}\right) \mathrm{d} X_{2}$,

where

$R_{\mathrm{S}}=\frac{r_{\mathrm{S}}}{\sqrt{\pi} R_{\mathrm{F}}} \frac{z_{0}}{z_{1}}$

is the reduced source radius which is close to the projected source radius onto the screen plane when $z_{1} \gg z_{0}$, expressed in units of $\sqrt{\pi} R_{\mathrm{F}}$. It appears that the diffraction patterns from the different parts of an extended source will be washed out if $R_{\mathrm{S}} \gg 1$ or, equivalently, if the angular radius of the source $\theta_{\mathrm{S}}$ is much larger than the angular Fresnel radius $\theta_{\mathrm{F}}$. This is why there is a possibility to observe a reasonably contrasted pattern only for extra-galactic sources, as shown in Table 1 . In this table, we have focused our attention on the hottest stellar classes that corresponds to the smallest stellar radii.

As an illustration, Fig. 4 shows the diffraction patterns for a source of reduced radius equal to 1,2 and 4. Figure 5 gives the maximum contrast of the pattern as a function of $R_{\mathrm{S}}$ for two values of the step size $\delta$. This shows the very strict limitations on the angular size of the target object to get a measurable contrast: $\theta_{\mathrm{S}}$ should not be too large with respect to $\theta_{\mathrm{F}}$.

Examination of Tables 1 and 2 shows that every star gives a contrasted diffraction pattern through the atmosphere if the optical path changes by the order of $\lambda$ at the $\mathrm{cm}$ scale. This happens for a star close to horizon, and explains the stronger twinkling contrast observed at this moment ${ }^{4}$. More interestingly, we note that only remote stars have a chance to give a reasonably contrasted fringe system on Earth through a Galactic screen (at $>300 \mathrm{pc}$ ). We will focus on two configurations: A small source located in a Magellanic Cloud (LMC or SMC) or a bigger source located in M31, with a screen located at a typical halo object distance $(\sim 10 \mathrm{kpc})$.

Numerical calculations show that the maximum contrast region has the reduced size of the source (see Fig. 4). The maximum intensity occurs when the source periphery is almost tangent to the step when seen from the observer's plane.

${ }^{4}$ When the stars are high in the sky, their twinkling originates in the weak scintillation regime described below. 


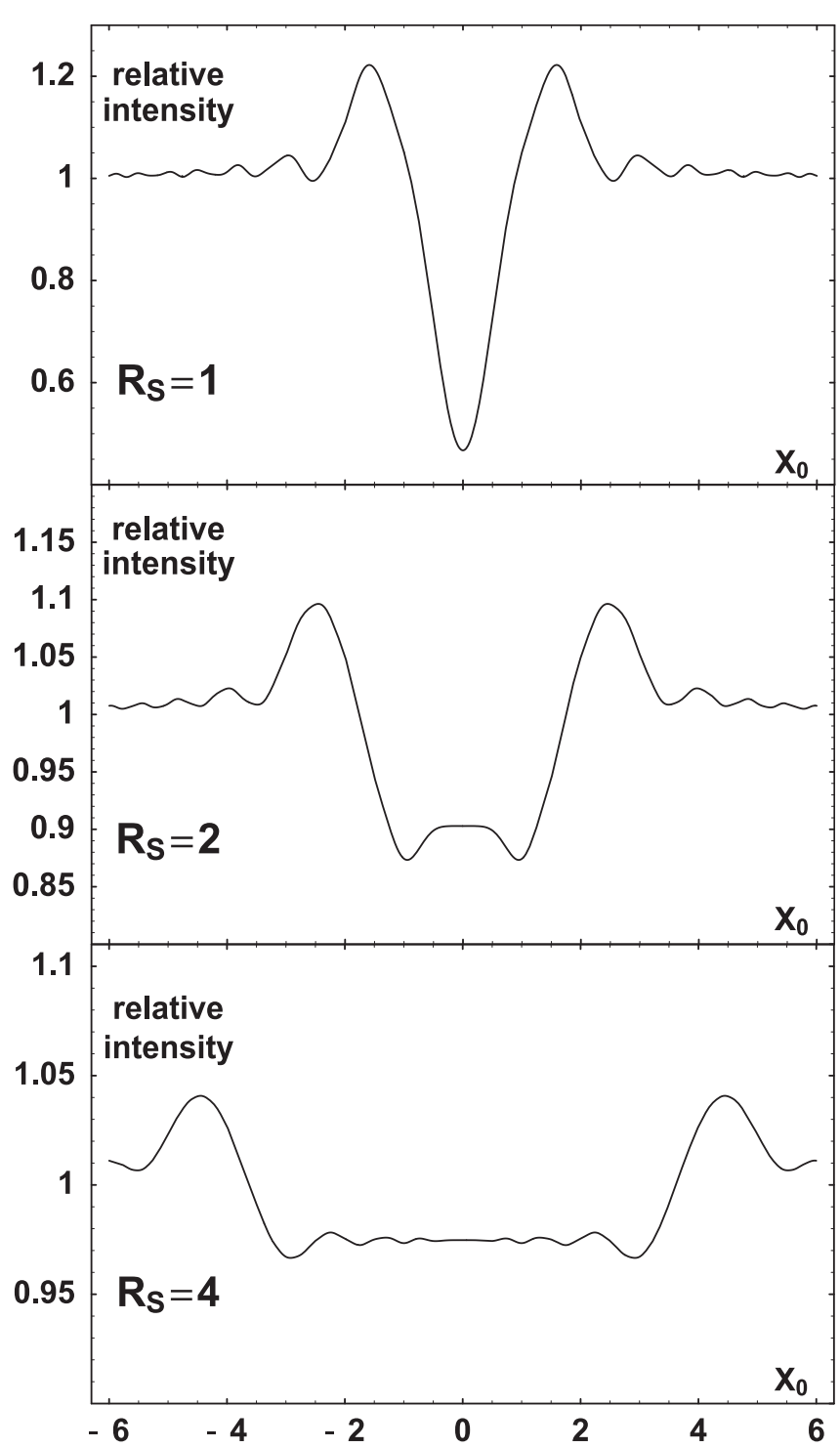

Fig. 4. Diffraction pattern produced perpendicularly to a step of optical path of $\delta=\lambda / 2$, for extended disk-sources of reduced radius $R_{\mathrm{S}}=1$ (top), 2 (middle) and 4 (bottom). The size of the central depression corresponds to the reduced source size. Notice that the vertical scales are very different because the contrast is much lower for $R_{\mathrm{S}}=4$.

When the step is out of the projected source disk, the interfringe is $\simeq 1.5 \times R_{\mathrm{F}}$, independently of the source radius.

\subsection{Chromatic source. Temporal coherence}

Up to now we have only considered monochromatic sources. The standard $U B V R I$ filter system has passbands that all satisfy $\Delta \lambda / \lambda<0.1$. With these filters, temporal coherence is sufficiently high to enable the formation of contrasted interferences between different parts of the wave-front, as long as the optical path difference is less than $\lambda^{2} / \Delta \lambda \sim 10 \times \lambda$. As this coherence length is large compared to the typical optical path differences we consider here, we can ignore the temporal coherence aspects in the following.

The inter-fringe scales with $R_{\mathrm{F}}$, i.e. with $\sqrt{\lambda}$, then $\Delta \lambda / \lambda<0.1 \Rightarrow \Delta R_{\mathrm{F}} / R_{\mathrm{F}}<0.05$. The contribution of the



Fig. 5. Fringe contrast as a function of the reduced star radius $R_{\mathrm{S}}$ $\left(\sim \theta_{\mathrm{S}} / \sqrt{\pi} \theta_{\mathrm{F}}\right.$ when the source is much farther from the observer than the screen), for 2 values of the step size: $\delta=\lambda / 4$ (thin line) and $\delta=\lambda / 2$ (thick line). For a large source radius, the contrast varies like $1 / R_{\mathrm{S}}$.

wavelength dispersion to the fringe jamming is then usually much smaller than the contribution due to the source extension in the configurations studied here.

\subsection{The observable phenomenon, summary}

Our study shows that an interference pattern with inter-fringe of $\sim R_{\mathrm{F}}(100-10000 \mathrm{~km})$ is expected on Earth when the line of sight of a sufficiently small astrophysical source (such as a remote star) crosses the edge of a structure that changes the optical path by a significant fraction of $\lambda$. Such structures move with respect to the line of sight with typical velocities given in Table 2. The observer's plane is then illuminated by an interference pattern moving with the same velocity (remember that the source is much farther than the screen). The shape of the interference pattern can also evolve, due to random turbulence in the scattering medium. We will base the present study on the assumption that the scintillation is mainly due to pattern motion rather than the pattern instability, as it is usually the case in radioastronomy observations (Lyne \& Graham-Smith 1998). The contrast of the pattern is critically limited by the angular size of the source; in the configurations proposed here, we expect a typical contrast ranging between $1 \%$ and $10 \%$. The time scale $t_{\text {scint }}$ of the intensity fluctuations is $t_{\text {scint }}=R_{\mathrm{F}} / V_{\mathrm{T}}$, where $V_{\mathrm{T}}$ is the transverse velocity of the structure. It is of order of $10-40 \mathrm{~s}$. As discussed just before, the inter-fringe scales with $\sqrt{\lambda}$; therefore, one expects a significant difference in the inter-fringe and in the time scale $t_{\text {scint }}$ between the red side of the optical spectrum and the blue side. This property might be used to sign the diffraction phenomenon at the $R_{\mathrm{F}}$ natural scale. We will see below that the $\lambda$ dependence is different if the 
Table 2. Examples of screen positions, corresponding Fresnel sizes, and fringing time scales at $\lambda=500 \mathrm{~nm}$. The typical relative velocity of the nearby stars is taken from the dispersions published by Cox (Allen) (2000).

\begin{tabular}{|l|c|c|c||c|c|c|}
\hline \hline Screen type & atmosphere & $\begin{array}{c}\text { solar } \\
\text { system }\end{array}$ & $\begin{array}{c}\text { solar } \\
\text { neighbourhood }\end{array}$ & $\begin{array}{c}\text { Galactic } \\
\text { thin disk }\end{array}$ & $\begin{array}{c}\text { Galactic } \\
\text { thick disk }\end{array}$ & $\begin{array}{c}\text { Galactic } \\
\text { halo }\end{array}$ \\
\hline Distance & $10 \mathrm{~km}$ & $1 \mathrm{AU}$ & $10 \mathrm{pc}$ & $300 \mathrm{pc}$ & $1 \mathrm{kpc}$ & $10 \mathrm{kpc}$ \\
Fresnel size & $2.8 \mathrm{~cm}$ & $109 \mathrm{~m}$ & $157 \mathrm{~km}$ & $860 \mathrm{~km}$ & $1570 \mathrm{~km}$ & $5000 \mathrm{~km}$ \\
\hline Angular size $\theta_{\mathrm{F}}(\mathrm{rad})$. & $2.8 \times 10^{-6}$ & $7.3 \times 10^{-10}$ & $5.1 \times 10^{-13}$ & $9.3 \times 10^{-14}$ & $5.1 \times 10^{-14}$ & $1.6 \times 10^{-14}$ \\
$\theta_{\mathrm{F}}$ in ${ }^{\prime \prime}$ & 0.6 & $1.5 \times 10^{-4}$ & $1.0 \times 10^{-7}$ & $1.9 \times 10^{-8}$ & $1.0 \times 10^{-8}$ & $3.3 \times 10^{-9}$ \\
\hline relative speed & $1 \mathrm{~m} \mathrm{~s}^{-1}$ & $10 \mathrm{~km} \mathrm{~s}^{-1}$ & $20 \mathrm{~km} \mathrm{~s}^{-1}$ & $30 \mathrm{~km} \mathrm{~s}^{-1}$ & $40 \mathrm{~km} \mathrm{~s}^{-1}$ & $200 \mathrm{~km} \mathrm{~s}^{-1}$ \\
time scale & $0.03 \mathrm{~s}$ & $0.01 \mathrm{~s}$ & $8 \mathrm{~s}$ & $29 \mathrm{~s}$ & $40 \mathrm{~s}$ & $25 \mathrm{~s}$ \\
\hline
\end{tabular}

screen produces optical path stochastic fluctuations at a length scale much smaller than $R_{\mathrm{F}}$.

\section{General discussion of the scintillation regimes}

Up to now, we have considered variations of the screen optical thickness at a scale close to $R_{\mathrm{F}}$. In the screen plane, $R_{\mathrm{F}}$ can be considered as a coherence domain, in the sense that - as mentioned earlier - integral (9) is dominated by the contribution of a region characterized by the Fresnel radius. This means that only the patch of the wavefront within a few Fresnel radii contributes coherently to the integral. The details of the optical path variations inside this domain then drive the diffraction pattern, leading to the (small scale) diffractive scintillation. If there is a large scale structure $\left(\gg R_{\mathrm{F}}\right)$ in the optical path variations, a succession of focusing and defocusing configurations occurs, where the first phase term $k \delta\left(x_{1}, y_{1}\right)$ in integral (9) can partially compensate or enhance the second phase term; the coherence domain that contributes to the integral can then become larger or smaller than $R_{\mathrm{F}}$. In the case of large optical path variations, several coherence domains can also converge and the average luminosity can be estimated through the geometrical optics approximation. The intensity then depends on the local focal length produced by the optical path variations on the screen. Intensity variations - called refractive scintillation - arise at a length scale larger than $R_{\mathrm{F}}$.

Intensity scintillation is well known in observations of compact radio sources. Indeed scintillation of radio-pulsars is used to study the nearby interstellar and solar system media. The large wavelength and the very small size of the sources allow one a good control of this technique. In this section, we will summarize the conditions and characteristics for the different scintillation regimes that occur in pulsar radio-observations, and adapt them to star optical observations. Extensive literature exists on pulsar physics. Reviews can be found in Lyne \& Graham-Smith (1998) and Narayan (1992); the scaling laws described below are taken and adapted for the latest reference.

The distinction between the regimes is based on the relative values of the length scale of the optical path fluctuations and of the Fresnel scale $R_{\mathrm{F}}$. The screen is characterized by its diffractive length scale $R_{\text {diff }}$, defined as the separation in the screen plane for which the root mean square of the optical path difference $\delta\left(x_{1}^{\prime}, y_{1}^{\prime}\right)-\delta\left(x_{1}, y_{1}\right)$ is $\lambda / 2 \pi$ (see Fig. 6). As the patch of the

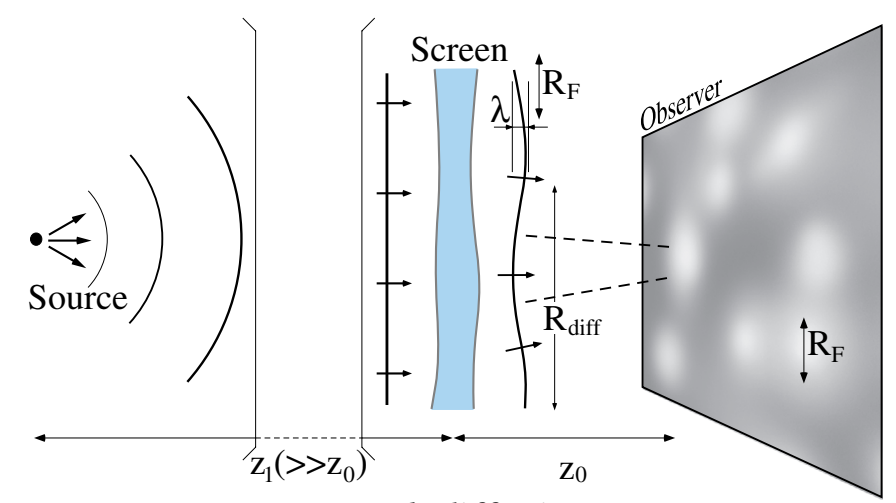

Weak diffusion

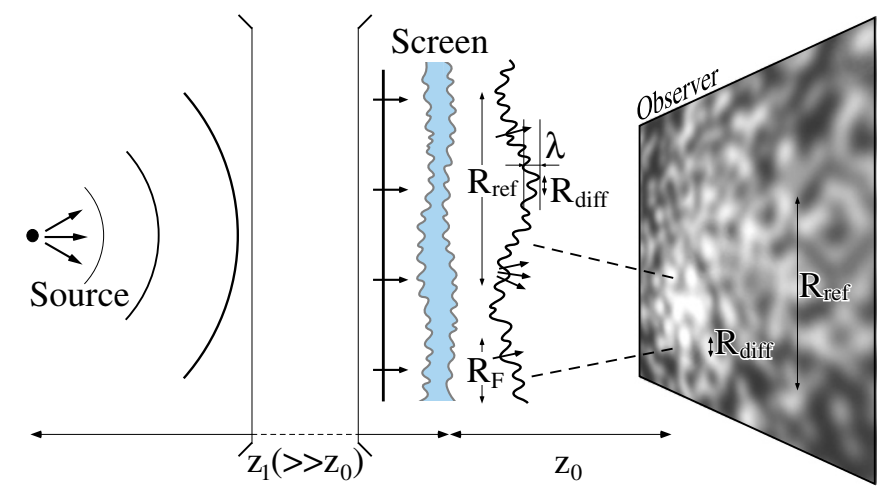

Strong diffusion

Fig. 6. The two scintillation regimes: sections of the wavefront after screen crossing. - Top panel: $R_{\text {diff }} \gg R_{\mathrm{F}}$. The weakly distorted wavefront produces a weak scintillation at scale $R_{\mathrm{F}}$ in the observer's plane. - Bottom panel: $R_{\text {diff }} \ll R_{\mathrm{F}}$. The strongly distorted wavefront produces strong scintillation at scales $R_{\text {diff }}$ (diffractive mode) and $R_{\text {ref }}$ (refractive mode) in the observer's plane.

wavefront contributing to integral (9) has a size of order of $R_{\mathrm{F}}$ in the screen plane, we can consider two very different situations since integral (9) has a completely different behaviour whether $R_{\text {diff }} \gg R_{\mathrm{F}}$ or $R_{\text {diff }} \ll R_{\mathrm{F}}$. 
Table 3. Conditions and characteristics of the different scintillation regimes for point-like and extended sources of apparent angular size $\theta_{\mathrm{S}}$. $R_{\text {scint }}$ is the characteristic length scale of the illumination pattern, and $t_{\text {scint }}$ is the characteristic time scale of the intensity variations for a screen moving at transverse speed $V_{\mathrm{T}}$ with respect to the line of sight. The modulation index $m_{\text {scint }}$ is the root mean square amplitude of the intensity scintillation. $m_{\text {scint }}$ depends only on the $\theta_{\text {diff }}$ to $\theta_{\mathrm{S}}$ ratio for the diffractive mode. For the other scintillation modes, $m_{\text {scint }}$ is given for stochastic fluctuations of the optical path length due to Kolmogorov turbulence in the scattering medium.

\begin{tabular}{l|c|c|c}
\hline \hline & $R_{\text {diff }} \gg R_{\mathrm{F}}$ & \multicolumn{2}{|c}{$R_{\text {diff }} \ll R_{\mathrm{F}}$} \\
& Weak scintillation & \multicolumn{2}{|c}{ Strong scintillation } \\
& & diffractive mode & refractive mode \\
\hline characteristic length & $R_{\mathrm{F}}$ & $R_{\text {diff }}$ & $R_{\text {ref }} \gg R_{\text {diff }}$ \\
characteristic angle & $\theta_{\mathrm{F}}$ & $\theta_{\text {diff }}$ & $\theta_{\text {ref }}$ \\
coherence passband $\Delta \lambda / \lambda$ & $\sim 1$ & $\left(R_{\text {diff }} / R_{\mathrm{F}}\right)^{2}$ & $\sim 1$ \\
\hline Point-source condition & $\theta_{\mathrm{S}}<\theta_{\mathrm{F}}$ & $\theta_{\mathrm{S}}<\theta_{\text {diff }}$ & $\theta_{\mathrm{S}}<\theta_{\text {ref }}$ \\
\hline$R_{\text {scint }}$ & $R_{\mathrm{F}}$ & $R_{\text {diff }}$ & $R_{\text {ref }}$ \\
$t_{\text {scint }}$ & $R_{\mathrm{F}} / V_{\mathrm{T}}$ & $R_{\text {diff }} / V_{\mathrm{T}}$ & $R_{\text {ref }} / V_{\mathrm{T}}$ \\
$m_{\text {scint }}$ & $\left(R_{\mathrm{F}} / R_{\text {diff }}\right)^{\frac{5}{6}} \ll 1$ & $\sim 1$ & $\left(R_{\text {diff }} / R_{\mathrm{F}}\right)^{\frac{1}{3}} \ll 1$ \\
\hline Extended source & $\theta_{\mathrm{S}}>\theta_{\mathrm{F}}$ & $\theta_{\mathrm{S}}>\theta_{\text {diff }}$ & $\theta_{\mathrm{S}}>\theta_{\text {ref }}$ \\
\hline$R_{\text {scint }}$ & $R_{\mathrm{F}}\left(\theta_{\mathrm{S}} / \theta_{\mathrm{F}}\right)$ & $R_{\text {diff }}\left(\theta_{\mathrm{S}} / \theta_{\text {diff }}\right)$ & $R_{\text {ref }}\left(\theta_{\mathrm{S}} / \theta_{\text {ref }}\right)$ \\
$t_{\text {scint }}$ & $\left(R_{\mathrm{F}} / V_{\mathrm{T}}\right)\left(\theta_{\mathrm{S}} / \theta_{\mathrm{F}}\right)$ & $\left(R_{\text {diff }} / V_{\mathrm{T}}\right)\left(\theta_{\mathrm{S}} / \theta_{\text {diff }}\right)$ & $\left(R_{\text {ref }} / V_{\mathrm{T}}\right)\left(\theta_{\mathrm{S}} / \theta_{\text {ref }}\right)$ \\
$m_{\text {scint }}$ & $\left(R_{\mathrm{F}} / R_{\text {diff }}\right)^{\frac{5}{6}}\left(\theta_{\mathrm{F}} / \theta_{\mathrm{S}}\right)^{\frac{7}{6}}$ & $\theta_{\text {diff }} / \theta_{\mathrm{S}}<1$ & $\left(R_{\text {diff }} / R_{\mathrm{F}}\right)^{\frac{1}{3}}\left(\theta_{\text {ref }} / \theta_{\mathrm{S}}\right)^{\frac{7}{6}}$ \\
\hline
\end{tabular}

\subsection{Weak scintillation regime}

If $R_{\text {diff }} \gg R_{\mathrm{F}}$, then the random fluctuations of the optical path are small compared to the wavelength within the Fresnel zone. The screen is then qualified as weakly diffusive. Only weak perturbations of the wavefront are expected, essentially from the focusing/defocusing effects of the optical path fluctuations within this Fresnel zone. In this regime, only the wavefront patch enclosed in the Fresnel radius plays a significant role. Indeed as variations of $\delta\left(x_{1}, y_{1}\right)$ are small over the Fresnel domain, $\delta\left(x_{1}, y_{1}\right)$ cannot significantly change the total phase in integral (9) when $\left(x_{1}-x_{0}, y_{1}-y_{0}\right)$ is close to the Fresnel circle.

An alternative point of view is to consider that the emerging wave is a combination of the initial plane wave plus weak amplitude perturbing waves with a random distribution of wave normals. For the observer, a point source located behind the screen would appear like a main spot, surrounded by low luminosity spots. The resulting intensity variations when the screen moves from one Fresnel-size zone to the next are weak, and their typical time scale is $R_{\mathrm{F}} / V_{\mathrm{T}}$, where $V_{\mathrm{T}}$ is the transverse velocity of the screen with respect to the observer-source line of sight. Table 3 gives the conditions to observe this scintillation regime and its characteristics.

\subsection{Strong scintillation regimes}

If $R_{\text {diff }} \ll R_{\mathrm{F}}$, then the random fluctuations of the optical path produce strong random phase changes within the Fresnel zone. The screen is considered as a strongly diffusive filter because the wavefront is strongly perturbed. From our alternative point of view, one can consider that the contribution of the initial plane is almost completely redistributed after crossing the screen. The point source seen by the observer through the screen would now appear like scattered spots with random intensities. In this situation, the intensity is expected to strongly vary at the sub-Fresnel scale. Moreover, large length-scale structures in the optical path variations produce average focusing/defocusing effects over domains much larger than the Fresnel size. Two scintillation modes are then to be distinguished:

\section{- Diffractive scintillation}

As the optical path variations are larger than $\lambda$ within the Fresnel radius, one expects a strongly contrasted fringe system (for a point-like source). By contrast with the simple case studied in Sect. 5, where no explicit length scale other than $R_{\mathrm{F}}$ was introduced, there is now a different scale of optical path variations $\delta\left(x_{1}, y_{1}\right)$ characterized by $R_{\text {diff. Then, }}$. when the screen is shifted from a position to another located at distance $R_{\text {diff }}$, the values of integral (9) are not correlated between the two positions. The resulting intensity variations when the screen moves are large, and their time scale is $R_{\text {diff }} / V_{\mathrm{T}}$.

As discussed in Sect. 5.2, diffractive scintillation is critically dependent on the source size. In contrast, the time scale does not depend on the wavelength $\lambda$, but the positions of the maxima/minima do, thus limiting the temporal coherence of the fringes. When $\lambda$ changes by $\Delta \lambda$, the fringes produced by $R_{\text {diff-size structures are displaced }}$ by $\sim z_{0} \Delta \lambda / R_{\text {diff. }}$. The fringe systems are decorrelated when this displacement is $\sim R_{\text {diff }}$, i.e. when $\Delta \lambda / \lambda \sim\left(R_{\text {diff }} / R_{\mathrm{F}}\right)^{2}$ (see Table 3).

\section{- Refractive scintillation}

This effect results from the strong stochastic variations of the optical path produced by large scale structures of the 


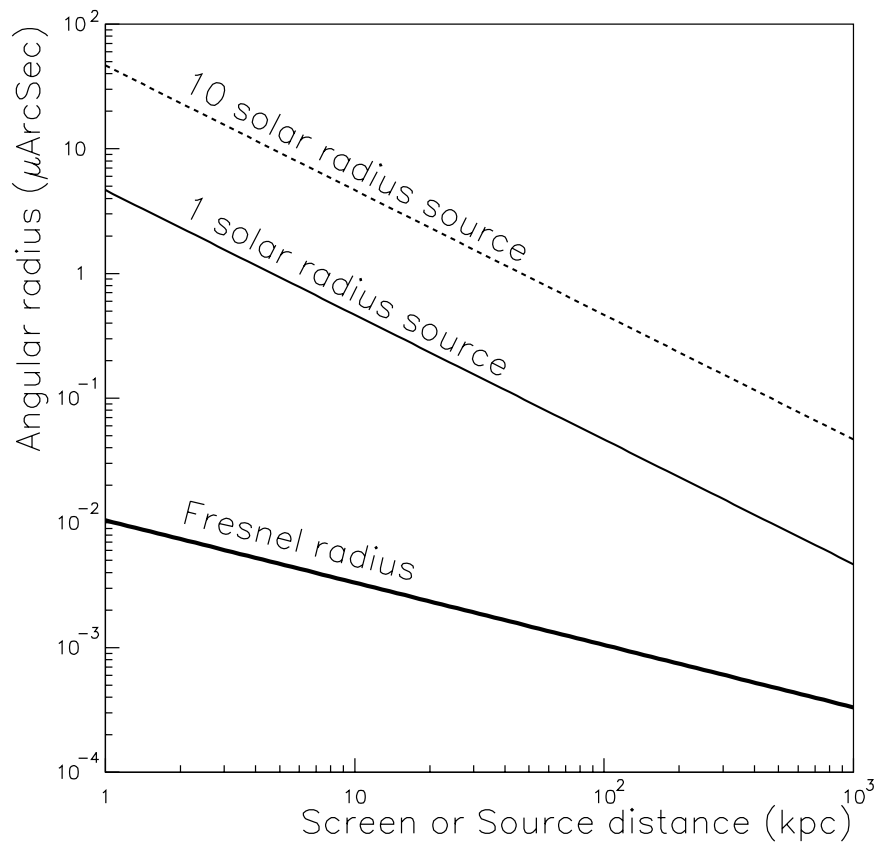

Fig. 7. Apparent stellar angular radius $\theta_{\mathrm{S}}$ for a solar-like star $\left(r_{\mathrm{S}}=r_{\odot}\right)$ and for a star with $r_{\mathrm{S}}=10 r_{\odot}$ as a function of the distance. The lower line gives the Fresnel radius $R_{\mathrm{F}}$ as a function of the screen's distance, illustrating the fact that the source has to be much farther than the screen to give contrasted diffractive scintillation.

screen. Let $R_{\text {ref }}$ be this additional length scale. In contrast to the case of weak scintillation, the large variations of the optical path can considerably change the values of the integrand phase of integral (9) at the limits of the Fresnel domain. Focusing/defocusing effects are not restricted to the Fresnel zone any more, and light from "other" Fresnel domains can be focused if the optical path variations are large enough. Such effects now involve regions of typical size defined by $R_{\text {ref }}$, and their time scale is $R_{\text {ref }} / V_{\mathrm{T}}$.

Source size is much less critical for the visibility of this mode. The time scale also does not depend on the wavelength and the illumination patterns are coherent within a wide passband (see Table 3).

The strong scattering diffraction mode is the most promising in terms of contrast, as demonstrated in Sect. 5. Figure 7 allows one to compare the apparent stellar angular size $\theta_{\mathrm{S}}$ with the natural angular Fresnel scale $\theta_{\mathrm{F}}$ at $\lambda=500 \mathrm{~nm}$. Figure 8 shows the iso-contrast lines in the fringing system expected in the case of strong diffractive scintillation. Expressed in practical units, the modulation index as defined in Table 3 is in this case

$m_{\text {scint }}=0.071\left[\frac{\lambda}{500 \mathrm{~nm}}\right]^{\frac{1}{2}}\left[\frac{R_{\mathrm{diff}}}{R_{\mathrm{F}}}\right]\left[\frac{r_{\mathrm{S}}}{r_{\odot}}\right]^{-1}\left[\frac{z_{0}+z_{1}}{1 \mathrm{kpc}}\right]\left[\frac{z_{0}}{1 \mathrm{pc}}\right]^{-\frac{1}{2}}$.

The example discussed in Sect. 5 corresponds to a diffractive scintillation regime that represents a transition between the weak and the strong regimes. The natural scale $R_{\mathrm{F}}$ is here the only relevant parameter depending only on the screen position and wavelength. For this transition regime, the modulation index estimated from the above formula is stronger than calculated in Sect. 5, because the approximations of Table 3 are not

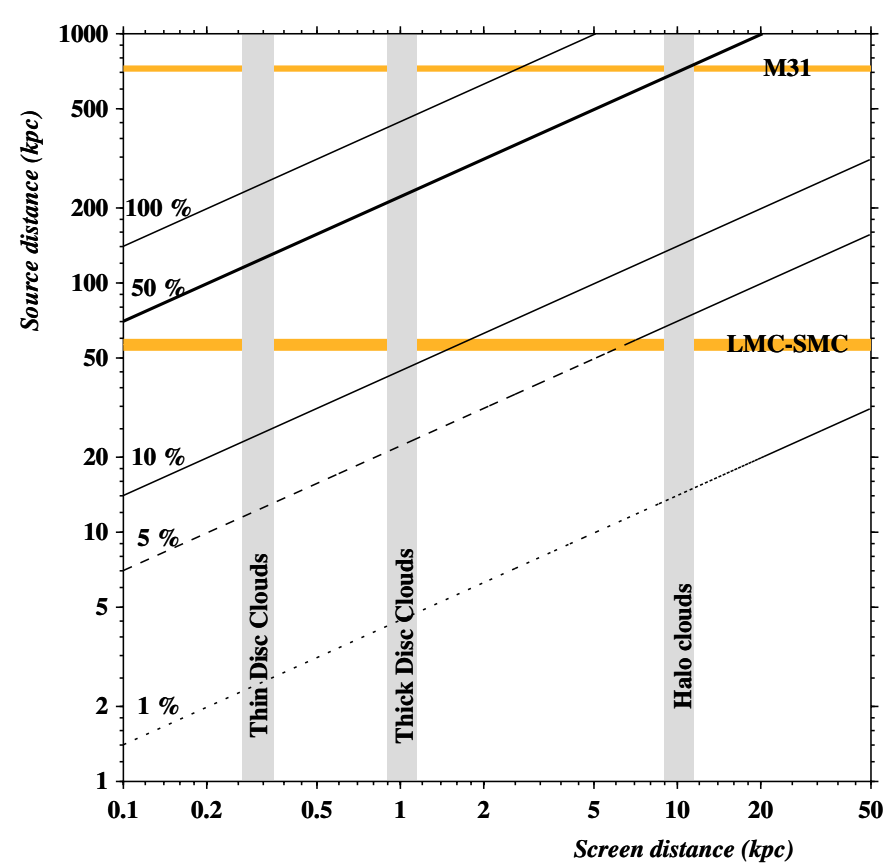

Fig. 8. Lines of equal diffractive scintillation modulation index at $\lambda=$ $500 \mathrm{~nm}$ in the source/screen distances plane. The modulation index is $100 \%$ (thick line), $50 \%, 10 \%, 5 \%$ and $1 \%$ (from top to bottom lines), assuming that the product $\left[\frac{R_{\mathrm{diff}}}{R_{\mathrm{F}}}\right]\left[\frac{r_{\mathrm{S}}}{r_{\odot}}\right]^{-1}$ is unity. This happens only for very small sources, and when $R_{\mathrm{diff}}$ is not too small compared to $R_{\mathrm{F}}$. Otherwise, the modulation index has to be multiplied by this product. Typical distances of disks and halo clouds are indicated, as well as the distances of the two most promising targets.

valid. For a B0V star in M $31\left(\theta_{\mathrm{S}}=4.7 \times 10^{-8}{ }^{\prime \prime}\right)$ and a screen at $1 \mathrm{kpc}\left(\theta_{\mathrm{F}}=1.0 \times 10^{-8}{ }^{\prime \prime}\right)$, the expected contrast for diffractive scintillation - setting $R_{\mathrm{diff}}=R_{\mathrm{F}}$ in the expression of Table $3-$ is $m_{\text {scint }} \sim\left(\theta_{\text {diff }} / \theta_{\mathrm{S}}\right) \sim\left(\theta_{\mathrm{F}} / \theta_{\mathrm{S}}\right)=0.21$, to be compared with the contrast of $\sim 0.1$ expected from a step of $\lambda / 2$ in the optical path (Fig. 5).

\subsection{Extreme scintillation}

For completeness, we should also mention an extreme scintillation regime, due to caustic effects, occurring when the observer approaches the focus of a refractive structure. Structures like the ones predicted by Combes \& Pfenniger (1997), with concentrations of diameter $D=10 \mathrm{AU}$ that produce a total variation in the optical path (from edge to center) $\Delta=50000 \times \lambda$ at $\lambda=500 \mathrm{~nm}$, should act as optical lenses. A naive focal length estimate gives $f \sim D^{2} / 8 \Delta=360 \mathrm{Mpc}$. The concentration power of such a lens is clearly much too weak to give a measurable signal on Earth. Moreover, the time scale of the intensity variations should be $t_{\text {scint }}=D / V_{\mathrm{T}}=(10 \mathrm{AU}) /\left(200 \mathrm{~km} \mathrm{~s}^{-1}\right)=$ 87 days. Other authors have discussed in detail the possible caustic effects with polytropic models of self-gravitating gas clouds, and the consequences on background star light-curves (Draine 1998; Rafikov \& Draine 2001). We will not consider this regime in the following. 


\subsection{Regime discrimination}

The appropriate tool to connect the screen structure with the scintillation features will be the temporal power spectrum of the intensity fluctuations. If strong diffractive and refractive regimes take place together, two peaks are expected in the power spectra. Their relative power will also be connected with the source size. The so-called inverse problem, i.e. the determination of a density fluctuation model of the interstellar medium from scintillation observations has been extensively discussed by radio-astronomers (Narayan 1988), but is beyond the scope of this exploratory paper.

We will mainly discuss here the perspectives of the diffractive scintillation regime. Nevertheless, we will also sometimes mention the refractive regime because important density fluctuations may occur at a much smaller scale than the global structure, due to gaseous turbulence phenomena.

\section{Possible configurations for detectable diffractive scintillation}

One of the main outcomes of the previous discussions is that only small angular sources may give a reasonably contrasted diffractive scintillation. We now focus on this diffractive process, because its modulation index can be large and is easy to predict with basically no hypothesis on the detailed screen structure. We will only assume that a regime characterized by $R_{\text {diff }} \leq R_{\mathrm{F}}$ can be established, or that at least a transitory regime as described in Sect. 5 - characterized by $R_{\mathrm{F}}-$ can occur, for example if an inhomogeneity due to a turbulent mechanism crosses the line of sight. Table 4 that combines data from Tables 1 and 2 lists some configurations that should produce diffractive scintillation and gives their characteristics. Depending on the diffusion strength, other regimes may take place; the reader may adapt the forthcoming discussion to these regimes, after rescaling the characteristic length, time and contrast according to Table 3. Optical depth may also be considerably larger in those other regimes because they are strongly dependent on the screen structure. We will essentially consider minimum optical depths in what follows.

To end the discussion about the screen configurations, we have to mention here that a diffusive screen located near the source, or in the galaxy of the source, do not produce scintillation, due to the size of the source. In such a configuration $\left(z_{1} \ll z_{0}\right)$, the approximation of an incident plane wave on the screen is not valid any more, and calculation should be redone starting with Eq. (8). The Fresnel zone is then defined by $R_{\mathrm{F}}=\sqrt{\lambda / 2 \pi\left(1 / z_{0}+1 / z_{1}\right)^{-1}} \sim \sqrt{\lambda z_{1} / 2 \pi}$ instead of $\sqrt{\lambda z_{0} / 2 \pi}$ (see e.g. Born \& Wolf 1975 or Sommerfeld 1954). The $z_{0} / z_{1}$ multiplier that enters the reduced radius expression (21) is very large; consequently, spatial coherence is completely lost in this situation. Moreover, a diffusive screen close to the source cannot increase significantly its apparent size for a simple geometrical reason: the image of a source is not significantly distorted by diffusion occurring near the emission point. We will ignore such effects in the following.

\section{Optical depths and event rates for diffractive and refractive scintillations}

In this section, we want to quantify the probability to observe scintillation produced by disk or halo molecular clouds. The $1 \%$ surface filling factor predicted in the model of Pfenniger \& Combes (1994) for gaseous structures is also the maximum optical depth for all the possible refractive (weak or strong) and diffractive scintillation regimes. Nevertheless, we want to consider here the pessimistic case where the optical depth for the strong regimes is much smaller: in the thick disk, the typical transverse speed of a structure is $40 \mathrm{~km} \mathrm{~s}^{-1}$. Then the typical crossing time of such a structure should be $\sim 400$ days. Under the hypothesis that strong diffractive regime is expected only when the structure enters or leaves the line of sight ${ }^{5}$, the duration for this regime is of order of $\sim 5 \mathrm{~min}$ (time to cross a few fringes). Then the optical depth $\tau_{\text {scint }}$ for such regime is at least of order of $10^{-7}$ and the average exposure needed to observe one event of $\sim 5$ min duration is $10^{6} \mathrm{star} \times \mathrm{hr}$.

If the gaseous structures belong to the Galactic halo instead of the thick disk, the same order of magnitude is also expected for the optical depth.

We will neglect the multi-diffusion eventuality, given the small sky fraction occupied by the structures. For our study, the case of a thick (longitudinally extended) screen needs a formalism adaptation only if the extension along the line of sight is such that $R_{\mathrm{F}}$ significantly changes within the structure. Several authors (Lyne \& Graham-Smith 1998; Tatarskii \& Zavorotnyi 1980) have studied very thick screens and found a similar behaviour to the one that prevails for thin screens.

\section{Feasibility of observations}

The diffractive regime with Galactic hidden $\mathrm{H}_{2}$ gas (see the three last columns of Tables 2 and 4) can happen with contrasts better than $1 \%$ only if the source has an angular size smaller than a few $\sim 10^{-12}$ rad. For a given surface temperature this constraint is equivalent to a constraint put on the source (star, $\mathrm{SN}$, quasar...) magnitude. Choosing objects with the highest surface luminosity in Table 4 indicates that the minimal magnitude of stars whose light is likely to undergo a few percent modulation index is about $M_{V}=20.5$. Therefore, diffractive scintillation search needs the capability to sample every $\sim 10 \mathrm{~s}$ (or faster) the luminosity of stars with $M_{V}>20.5$, with a point-to-point precision better than a few percent. This performance can be achieved using a 2 meter telescope with a high quantum efficiency detector.

Indeed, the optimal relative photometric precision $\Delta$ is related to the star magnitude $M$, the exposure time $T_{\text {exp }}$, the seeing, the sky background magnitude $\mu$ per $\operatorname{arcsec}^{2}$ and the

\footnotetext{
5 The validity of this hypothesis as well as the optical depth estimate for each scintillation regime depend on the internal structure model. Turbulence or any process creating filaments, cells, bubbles or fluffy structures should be taken into account.
} 
Table 4. Configurations leading to strong diffractive scintillation. Numbers are given for $\lambda=500 \mathrm{~nm}$. Interstellar absorption is not taken into account for the magnitude estimates.

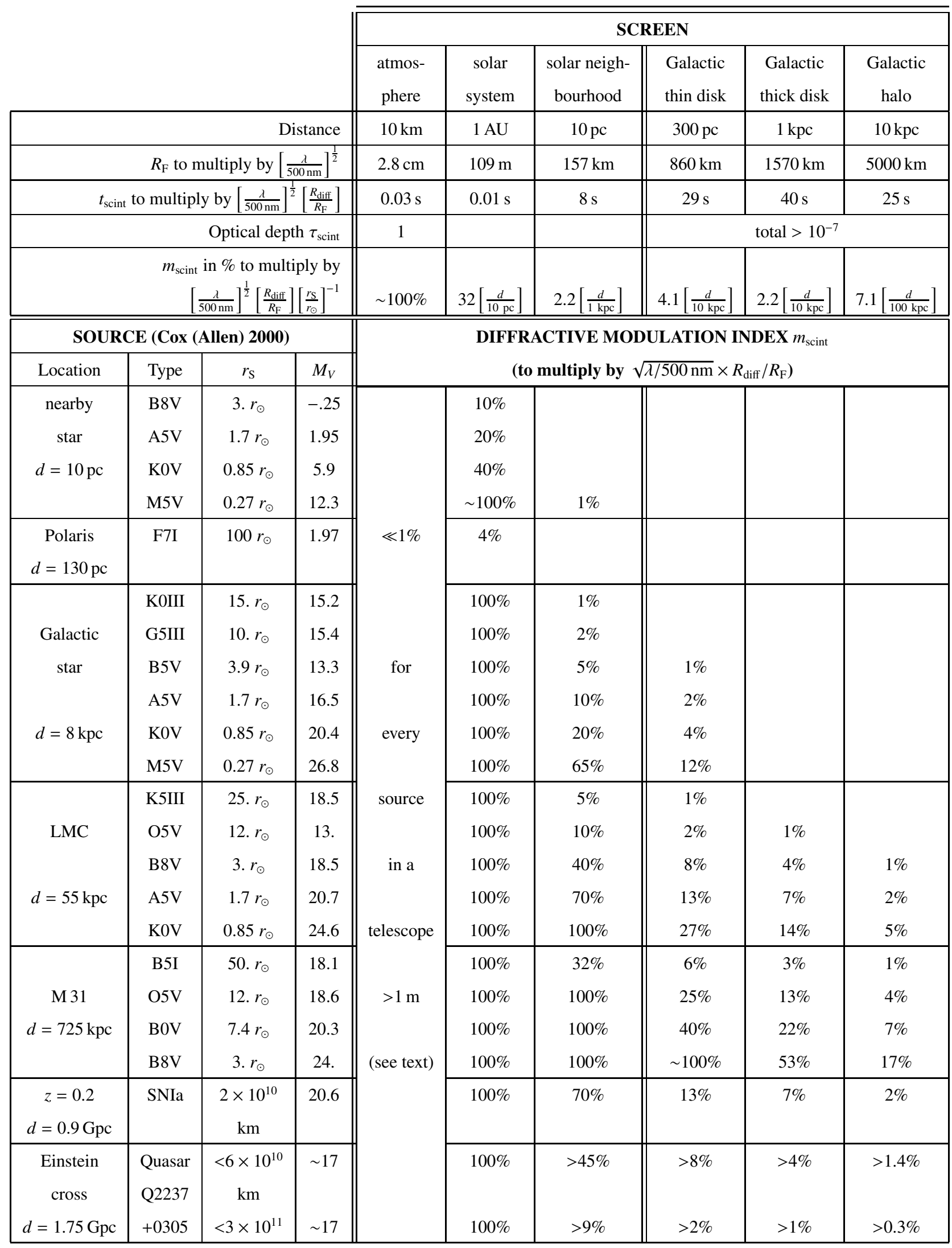


telescope collecting surface $S_{\text {tel }}=\pi D_{\text {tel }}^{2} / 4$ through the following formula, taken from (Moniez \& Perdereau 2001):

$$
\begin{aligned}
T_{\exp } \simeq & \frac{10^{M / 2.5}}{S_{\text {tel }} \Phi(M=0)} \frac{1}{\Delta^{2}} \\
& \times\left[0.27+1.5 \sqrt{0.25+1.13\left[\frac{\text { seeing }}{1^{\prime \prime}}\right]^{2} 10^{\frac{M-\mu}{2.5}}}\right]^{2},
\end{aligned}
$$

where $\Phi(M=0)$ is the photo-electron flux collected at observatory level by the detector in the considered passband per $\mathrm{m}^{2}$ collecting surface, for a $M=0$ star. This relation is valid as long as the readout noise remains negligible. For a telescope with two reflectors of 0.87 transmission each, equipped with a high quantum efficiency detector $(\sim 90 \%), \Phi\left(M_{V}=0\right) \sim$ $4.2 \times 10^{9} \gamma \mathrm{e}^{-} / \mathrm{s} / \mathrm{m}^{2}$ in the $V$-band. For good observation conditions (seeing $=1^{\prime \prime}, \mu>21.7$, i.e. moon-less nights), the squared term of expression (23) is $\sim 2$ for stars with $M_{V}=20.5$, and negligibly smaller for somewhat brighter stars. Then the relation simplifies into

$$
\Delta \simeq 30 \% \times 10^{\frac{M_{V}-20.5}{5}} \times \frac{1}{\sqrt{T_{\exp }(\mathrm{s})}} \times \frac{1}{D_{\text {tel }}(\mathrm{m})},
$$

where $D_{\text {tel }}$ is the diameter of the telescope. The photometric precision $\Delta$ would be $5 \%$ with $10 \mathrm{~s}$ exposures on a $M_{V}=20.5$ star, with a 2 meter telescope. Neglecting the interstellar absorption, this is sufficient to detect the most contrasted (and the longest) diffractive scintillation events, as can be seen in Table 4.

We wish to stress that the continuity of the monitoring is not a critical issue. As events last only for a few minutes in our scenario, the detection efficiency will not be affected by data taking interruptions, contrary to the case of microlensing searches. The essential parameters for the sensitivity of a detection setup will be the integrated exposure ${ }^{6}$ in star $\times$ hr, the sampling rate and the photometric precision.

The variation of the diffractive scintillation pattern with the wavelength $\lambda$ should also be used as a signature of the process. As emphasised in Sect. 6.2, if $R_{\text {diff }} \ll R_{\mathrm{F}}$ the time scale does not depend on $\lambda$, but the phase (the timing of extrema) changes with $\lambda$. In the particular case where $R_{\text {diff }} \sim R_{\mathrm{F}}$, the peak of the temporal power spectrum is expected to scale with $\sqrt{\lambda}$. For these reasons, multi-wavelength detection capability is highly desirable. The most powerful approach would be to get star spectra at a high time sampling rate. The equivalent in radioastronomy is called the dynamic spectrum, showing the intensity fluctuations in a 2D (time versus wavelength) diagram (see for example Gupta et al. 1994).

According to Table 4, M31 hot main sequence stars are the most promising targets, but LMC and SMC small stars are probably easier to distinguish from their surroundings.

\footnotetext{
${ }^{6}$ If dead-times larger than a few minutes occur, they should be subtracted from the exposure because events can be contained and subsequently lost in such time intervals. This is also a noticeable difference from the microlensing searches.
}

\section{Foreground effects, background to the signal}

One of the challenges to extract an interstellar intensity scintillation signal is the disentangling of the scintillation due to the foreground media (atmosphere, solar system and nearby medium).

\subsection{Atmospheric intensity scintillation and absorption}

Atmospheric intensity scintillation should be easier to handle than naively expected. Dravins et al. (1997-98) report extensive studies of this phenomenon, that is connected with seeing studies. When short length scale interference patterns (a few $\mathrm{cm}$ ) produced in the diffractive mode enter a large aperture telescope, the collected light is averaged, since the aperture acts like a low pass filter. The modulation index is then much smaller than $1 \%$ for a telescope diameter larger than one meter. Moreover, the time scale of this diffractive regime is two orders of magnitude faster than the searched Galactic signal. As far as the weak intensity scintillation is concerned, it also induces very small modulation index $m_{\text {scint }}$ at $1-10 \mathrm{~s}$ time scale; the following formula gives the low-frequency component of intensity scintillation at optical wavelengths, at altitude site $h$ (Dravins et al. 1997-98, part III):

$m_{\text {scint }}\left(t_{\exp }\right) \sim 0.09 \frac{D_{\text {tel }}^{-\frac{2}{3}}}{\sqrt{2 t_{\text {exp }}}} \cos (Z)^{-1.75} \mathrm{e}^{\frac{-h}{8000 \mathrm{~m}}}$,

where $\cos (Z)^{-1}$ is the airmass and $t_{\exp }$ is the integration time. For a 2 meter telescope, an airmass of 2 and an observatory altitude of $2400 \mathrm{~m}$, we find that $m_{\text {scint }}(1 \mathrm{~s})=3 \times 10^{-3}$ and $m_{\text {scint }}(10 \mathrm{~s})=10^{-3}$. Thus intensity variations due to atmosphere will not seriously affect a diffractive interstellar scintillation signal of $1 \%$ amplitude or more.

Any other long time scale atmospheric effects such as absorption changes at the sub-minute scale (due to fast cirruses for example) should be easy to remove as long as nearby stars are monitored together. At this time scale, the light of stars within a small angular distance undergo the same atmospheric effects at the same time, or with a short delay. A careful subtraction of such collective effects should in principle considerably reduce this source of background.

\subsection{Intensity scintillation due to the solar system medium, and to solar neighbourhood structures}

\section{- Interplanetary medium: from 0 to $5 \times \mathbf{1 0}^{-6}$ pc}

As can be seen Table 4, diffractive scintillation produced by interplanetary medium have a characteristic time of $10^{-2} \mathrm{~s}$, therefore it does not disturb an hypothetic Galactic gas signal.

The eventuality of a slow refractive regime has to be examined in more details: Studies of the atmospheric properties by (Dravins et al. 1997-98, part I) were mainly done with Polaris star. This star is far enough $(130 \mathrm{pc})$ to scintillate through interplanetary screens. Unfortunately, this star is big $\left(\sim 100 r_{\odot}\right)$ and its light cannot be subjected to 
modulations of more than $4 \%$ in the diffractive regime ${ }^{7}$. Nevertheless, in the hypothesis of a refractive regime that we are discussing here, we know that this not very critical, and Polaris measurements should then really be a reliable indicator of this latter scintillation type. As the measured modulation index of this star is much smaller than $1 \%$ at any frequency, this gives us a good preliminary indication that both diffractive and refractive scintillations from interplanetary medium are probably negligible toward Polaris direction.

\section{- Solar neighbourhood: from $5 \times 10^{-6}$ to $10 \mathrm{pc}$}

This conclusion also applies to solar neighbourhood screens (up to $\sim 10 \mathrm{pc}$ ) for the refractive regime, but the size of Polaris prevents us from drawing any conclusion relative to the diffractive regime.

The solar system is embedded in a local interstellar cloud extending not farther than $10-20 \mathrm{pc}$. The average column density of atomic hydrogen through this structure is less than $10^{19} \mathrm{~cm}^{-2}$ towards the Galactic center (Lallement et al. 1995; Ferlet 1999), which is negligible compared with the column density of the molecular clouds we are looking for. If molecular or atomic overdensities occur in this local region - typically at $10 \mathrm{pc}-$, then according to Table 4 , many types of stars located at $\sim 8 \mathrm{kpc}$, including red giants, should undergo a contrasted diffractive scintillation; as it can be deduced from this Table 4, the distinctive feature of scintillation through more distant screens $(>300 \mathrm{pc})$ is that only the smallest stars are expected to scintillate. It follows that a strategy consisting in the simultaneous monitoring of many different types of stars located at different distances should allow one to discriminate effects due to solar neighbourhood gas and due to more distant gaseous structures.

\section{- The local interstellar medium: from 10 to 200 pc}

The local interstellar cloud itself is located inside a bubble, which is a $50-200$ pc cavity mainly containing hot ionized gas. Ionized hydrogen has a negligible effect on the visible light propagation. The remaining atomic plus molecular hydrogen components contribute for a total column density of less than $10^{20} \mathrm{~cm}^{-2}$ up to $200 \mathrm{pc}$ (Lehner et al. 2003; Lallement et al. 2003). This is again much smaller than the column density due to molecular clouds. If dense atomic or molecular structures are located at such distances or beyond, they start to enter our domain of interest.

\subsection{Possible sources of fake signal?}

There is no known physical process that can produce $>1 \%$ intrinsic intensity variations of an ordinary star at the minute time scale. Asterosismology involves acoustic modes that produce a few tens of ppm intensity variations in the "high" frequency domain of a few minutes (Christensen-Dalsgaard 1998). Planet transit could also give a fast luminosity change, but are also expected to exhibit intensity variations smaller than $0.1 \%$. Granularity of the star surface, spots or eruptions would

${ }^{7}$ Polaris is also a spectroscopic binary system that may complicate the interference system. induce much lower frequency intensity variations than the diffractive scintillation. A few categories of recurrent variable stars exhibit important emission variations at the minute time scale (Sterken \& Jaschek 1996). Among them are the rare types UV Ceti and flaring Wolf-Rayet stars. Both types are easy to identify from their spectral characteristics. UV-Ceti are also very faint stars (absolute magnitude $>15$ ) and only the closest ones could contaminate a monitoring sample.

\subsection{Technical limitations}

Blending of stars, which is common in the LMC/SMC/M 31 crowded fields, may attenuate the measured contrast of a fringed pattern. Careful simulations are needed to measure the impact of these limitations. Adaptative optics or space measurements are possible ways to reduce this impact. Another technical difficulty is the risk of getting complicated (and fluctuating) point spread functions as a result of the very short exposures. Such circumstances could make necessary the use of performant subtraction algorithm, given the very crowded environment.

\section{Observation specifications and strategy}

A careful feasibility study should be made before starting an ambitious observation program: The stability of light curves of nearby stars should be checked with a low cost telescope at the sub-minute time scale, to test the control of collective intensity fluctuations due to meteorological phenomena and to explore possible limitations due to interplanetary gas. After such preliminary studies, an observational program could start with the specifications described below. These specifications are optimized for the search for diffractive scintillation in a large sample of stars. We showed in Table 4 that supernovæ and "small" quasars ${ }^{8}$ may also be subjected to diffractive scintillation; but the possible smallness of the optical depth $\left(\sim 10^{-7}\right)$ and the scarcity of such sources probably don't make them suitable targets for the first searches of diffractive scintillation. Moreover, one should not forget that diffractive scintillation of such sources is not expected from clouds located far beyond the Galaxy (see end of Sect. 7). Conversely, refractive regimes, which may have much larger optical depth, could be searched on any target.

\subsection{From Earth, with a single telescope}

- Optics

As already mentioned, at least a 2 meter class telescope is required to search for scintillation in $<10 \mathrm{~s}$ exposures on $20<M_{V}<21 \mathrm{LMC} / \mathrm{SMC}$ or M 31 stars.

First order adaptative optics will lead to a better photometric precision, but second-order adaptative optics (that corrects focusing/defocusing effects of atmosphere) is probably not really necessary since the atmospheric intensity scintillation should be negligible.

8 The Einstein's cross Q2237+0305 considered in the tables is the quasar that has the most precise size determination. It is also located at an optimal redshift $z=1.695$, corresponding to the maximum possible angular distance according to the currently published cosmological parameters, and then to a minimal angular size $\theta_{\mathrm{S}}$. 


\section{- Filters}

Monitoring in infra-red wavelength is not clearly a decisive advantage; the Fresnel radius is larger, but red stars are also usually larger. In contrast, monitoring stars with at least two different passbands should help to disentangle diffractive scintillation from the refractive one. In the case of diffractive scintillation, the phase differences of the fringe systems between two different passbands will provide constraints on the ratio of the screen distance to the diffusion scale $R_{\text {diff }}$ (see Sect. 6.2).

- Detector

A fast, low-background readout detector is essential to make possible the requested high sampling rate and to maintain a decent useful to dead time ratio. A possible option could to put an array of small (high quantum efficiency) frame-transfer CCDs at the focus of the telescope, that could be rapidly read (because they are small) in parallel during the exposures.

An alternative could be to use a series of very narrow CCDs (short columns), with continuous slow readout of the lines, to collect continuous light-curves without any dead-time. Such continuous readout option is not viable with a normal (not narrow) CCD, because of the light-curves mixing due to field crowding.

- Targets and fields

Imaging $1 \mathrm{deg}^{2}$ field on isophot $M_{V}=23 \mathrm{mag} / \mathrm{arcsec}^{2}$ towards LMC/SMC/M 31 allows one to monitor of order of $10^{5}\left(20<M_{V}<21\right)$ stars $^{9}$ (Elson et al. 1997; Hardy 1978). It is essential to choose fields containing stars with radii spanning a wide interval, and also containing a sample of nearby stars. This is necessary to extract information about the screens through the relationship between the temporal power spectrum and the source size and distance. In particular, nearby stars should provide a control sample of non-scintillating light-curves.

- Telescope time

Exposure of a few $10^{7} \mathrm{star} \times \mathrm{hr}$ (on small stars with $20<M_{V}<21$ ) during dark nights would provide a significant sensitivity to the existence of Galactic $\mathrm{H}_{2}$ structures. This needs typically one season of observations using a wide field telescope $\left(1 \mathrm{deg}^{2}\right)$ or a few seasons with a standard field telescope. Nevertheless, as already mentioned, there is no need for consecutive telescope time, making such program relatively flexible. Every sequence of consecutive images - longer than a few minutes - will contain its own events and will then be autonomous. Distinct but possibly simultaneous campaigns with different airmasses could certainly improve the knowledge of atmospheric effects.

- Complementary observations

In case of positive detection, complementary observations should be planned for scintillating candidate objects. The first requirement is the identification of the stellar type through spectroscopy, in order to check that the candidate do not belong to the very specific cataclysmic objects

\footnotetext{
${ }^{9}$ In more luminous fields like in the LMC bar, these stars would be blended by more luminous ones.
}

mentioned in Sect. 10.3, and to get an estimate of the distance and radius of the candidate. Such estimates are necessary to constrain the maximum distance of the screen. In the case of a star appearing to scintillate for a long time, time-resolved spectroscopy or multi-wavelength data taking as well as multiple detection (see below) will allow one to check the hypothesis of diffractive or refractive scintillation.

\subsection{From Earth: Array of telescopes}

A 2D array of telescopes, a few hundred and/or thousand kilometers apart, would certainly be the most powerful system to measure the characteristics of a diffusive screen: One single telescope will only provide a degenerated information (the time scale $t_{\text {scint }}=R_{\text {diff }} / V_{\mathrm{T}}$ ). Sampling a diffraction pattern with a $2 \mathrm{D}$ array would allow one to separately measure the geometrical scale $R_{\text {diff }}$ and the speed of the pattern. Atmospheric effects will be decorrelated between the telescopes, as well as the interplanetary gas effects. Nearby gaseous structures $(\sim 10 \mathrm{pc})$ are expected to produce $\sim 150 \mathrm{~km}$ fringes on Earth and Galactic structures are expected to produce $>1000 \mathrm{~km}$ fringes, that should be easy to distinguish with a handful of synchronized telescopes sampling a few thousand kilometers wide pattern. The speed and direction of the diffraction pattern (drift due to the relative velocity of the screen with respect to the line of sight), as well as the pattern's variations (due to the dynamics of the scattering medium), should also be measurable through the analysis of time delay between the telescopes. Obviously, any intrinsic star variability will be unambiguously identified, as it does not produce an intensity geometrical pattern.

\subsection{From space}

The main advantages of a space mission would be the better photometric precision and especially the much better spatial resolution, allowing to seriously reduce blending problems. As the intensity scintillation due to atmosphere is not critical at the level of $1 \%$, the cancellation of this specific scintillation in a spatial observatory will be interesting only for very weak scintillation searches. Among the operational spatial observatories and the planned missions, the HST observatory and COROT mission have been examined:

- COROT mission (Baglin et al. 2000) plans to monitor stars with a short time-scale sampling, but $1 \%$ precision photometry on $M_{V}=20$ stars in 10 s exposures is definitely unaccessible due to the modest size of the telescope.

- The Space Telescope Imaging Spectrograph instrument (STIS) of the Hubble Space Telescope (HST) is the only instrument that is able to offer a cycle time of $\sim 20 \mathrm{~s}$ for time-resolved spectroscopy or photometry; but this mode is only available on a sub-array of the CCD (Proffitt et al. 2002). Regrettably, only one (very) wide band filter $(550-1100 \mathrm{~nm})$ can be used to get $\sim 5 \%$ photometric precision in $10 \mathrm{~s}$ exposure time on a $M_{V}=20.5$ B0V star. This very wide band filter would already limit the temporal coherence, thus decreasing the diffractive 
scintillation contrast. The other filters have too narrow passbands to enable short exposures. Nevertheless, the HST angular resolution would be extremely valuable for monitoring M 31 fields, and there could be an interesting opportunity to further investigate. The other HST instruments have prohibitive overhead times.

\section{Data flow and analysis}

Data flow and analysis problems can be considered as the result of an hybrid of the EROS survey - for the massive photometric reduction - and the VIRGO gravitational wave experiment. For example, robust filtering developed in VIRGO for burst searches (Arnaud et al. 2003) should be appropriate to the search for an oscillating signal within a definite short period. The time scales of the data flow and of the signal are just multiplied by five orders of magnitude with respect to VIRGO.

\section{Studies to be done, developments and perspectives}

A simulation of the Galactic $\mathrm{H}_{2}$ gas distribution, and - more important - of the intra-cloud turbulence is clearly needed to produce more quantitative predictions on the optical depth, on the signal shape and on the temporal power spectrum.

Examination of the inverse problem will lead to more precise ideas on the information about the screen and the source that could be extracted from a statistical analysis based on the temporal power spectra. For example, as there is a clear connection between the diffractive pattern, the fringe contrast and the source size, the scintillation process may also be useful to improve the stellar radii knowledge, and to constrain supernovae and quasar dimensions.

It has been amply demonstrated that the angular size of the source is the critical parameter for the fringe contrast. Observations with a very large telescope would make possible the monitoring of stars much more distant than M 31, thus providing more contrasted diffractive intensity scintillation effects.

Existing data sets may allow the extraction of interesting constraints on the refractive scintillation mode. For instance EROS1 experiment produced 20000 photometric measurements of $\sim 100000$ stars in the LMC bar (Aubourg et al. 1995). $12 \mathrm{~min}$ blue and $8 \mathrm{~min}$ red exposures were alternatively taken. As refractive scintillation mode is much less sensitive to the source size than diffractive scintillation mode, the sources monitored by EROS1 were likely to scintillate. As this refractive mode could produce long time scale variations (longer than a few minutes), the EROS1 data set potentially contains interesting events.

As a final remark, one should also consider that the systematic survey of thousands of extra-galactic stars at high frequency $(\sim 0.1 \mathrm{~Hz})$ as proposed in this paper may give rise to surprises.

\section{Conclusions}

The minimal condition to observe an extra-galactic star scintillating through a Galactic molecular cloud is the existence of a non vanishing second order derivative of the optical path in the transverse plane. To fulfil this condition in the optical domain, stochastic column density fluctuations of $10^{19}$ molecules $/ \mathrm{cm}^{2}$ on the $10^{3}-10^{4} \mathrm{~km}$ length scale should take place, that produce optical path fluctuations of a fraction of a wavelength. It follows from existing models of molecular extended objects that this corresponds to column density relative fluctuations of a few ppm per $10^{3}-10^{4} \mathrm{~km}$ of transverse distance, of the same order than the average gradient.

Observations show that structuration of matter is present at all scales, and certainly do not refute the eventuality of stochastic fluctuations producing diffractive scintillation.

In this paper, we showed that there is an observational opportunity which results from the subtle compromise between the arm-lever of interference patterns due to hypothetic diffusive objects in the Milky-Way and the size of the extra-galactic stars.

The hardware and software techniques required for such observations are currently available. Tests are under way to validate some of the concepts described here. If no technical obstacle arises, there is a true opportunity to investigate such effects.

Acknowledgements. The preparation of this paper has benefited from fruitful discussions and interactions with: R. Ansari, M.A. Bizouard, E. Falgarone, J. Haissinski, P. Hello, N. Palanque-Delabrouille, S. Rahvar and P. Schwemling. Special thanks to J. Haissinski whose remarks allowed to considerably improve the manuscript.

\section{References}

Afonso, C., Albert, J. N., Andersen, J., et al. (EROS coll.) 2003, A\&A, 400,951

Alcock, C., Allsman, R. A., Alves, D. R., et al. (MACHO coll.) 2000, ApJ, 542, 281

Arnaud, N., Barsuglia, M., Bizouard, M.-A., et al. 2003, Phys. Rev. D, 67, 062004

Atsunori, Y. 2001, ApJ, 548, L127

Aubourg, E., Bareyre, P., Brehin, S., et al. (EROS coll.) 1995, A\&A, 301,1

Baglin, A., Appourchaux, T., Garrido, R., \& Weiss, W. W. 2000, The COROT Challenge, ESO F2/F3 proposal

Bennett, C. L., Halpern, M., Hinshaw, G., et al. (WMAP coll.) 2003, ApJS, 148, 1

Born, M., \& Wolf, E. 1975, Principles of Optics (Pergamon Press)

Caldwell, J., \& Coulson, I. 1986, MNRAS, 218, 223

Christensen-Dalsgaard, J. 1998, Lecture notes on Stellar Oscillations, Institut for Fysik og Astronomi, Aarhus Universitet

Combes, F., \& Pfenniger, D. 1997, A\&A, 327, 453

Cox, A. N. 2000, Allen's Astrophysical Quantities, 4th ed. (Springer)

CRC Handbook of Chemistry and Physics, 79th ed. 1998-1999

De Paolis, F., Ingrosso, G., Jetzer, Ph., \& Roncadelli, M. 1995, Phys. Rev. Lett., 74, 14

De Paolis, F., Ingrosso, G., Jetzer, Ph., \& Roncadelli, M. 1998, ApJ, 500, 59

Draine, B. T. 1998, ApJ, 509, L41

Dravins, D., Lindegren, L., Mezey, E., \& Young, A. T. 1998, Pub. of the Ast. Soc. of the Pacific, 1997, 109 (parts I and II), 110 (part III) 
Elson, R. A. W., Gilmore, G. F., \& Santiago, B. X. 1997 [astro-ph/9705149]

Ferlet, R. 1999, A\&A Rev., 9, 153

Filippenko, A. V. 1997, ARA\&A, 35, 309

Gupta, Y., Rickett, J., \& Lyne, A. G. 1994, MNRAS, 269, 1035

Hamilton, J. 1960, in Prog. in Nucl. Phys., 8, ed. O. R. Frisch (Pergamon Press)

Hardy 1978, Pub. of the Astron. Soc. of the Pacific 90, 132

Lallement, R., Ferlet, R., Lagrange, A. M., Lemoine, M., \& Vidal-Madjar, A. 1995, A\&A, 304, 461

Lallement, R., Welsh, B. Y., Vergely, L., Crifo, F., \& Sfeir, D. 2003, A\&A, 411, 447

Lasserre, T., Afonso, C., Albert, J. N., et al. (EROS coll.) 2000, A\&A, 355, L39

Lehner, N., Jenkins, E. B., Gry, C., et al. 2003, ApJ, 595, 858

Lyne, A. G., \& Graham-Smith., F. 1998, Pulsar Astronomy (Cambridge University Press)

Moniez, M., \& Perdereau, O. 2001, LAL report 01-71 [astro-ph/0110448]
Narayan, R. 1988, In Radio wave scattering in the interstellar medium, ed. J. M. Cordes, B. J. Rickett, \& D. C. Backer (New York: American Institute Physics), 17

Narayan, R. 1992, Phil. Trans. R. Soc. Lond. A, 341, 151

Olive, K. A., Steigman, G., \& Walker, T. P. 2000, Phys. Rep., 333, 389

Pfenniger, D., \& Combes, F. 1994, A\&A, 285, 94

Primack, J., Seckel, D., \& Sadoulet, B. 1988, Ann. Rev. Nuc. Part. Sci., B38, 751

Proffitt, C., et al. 2002, STIS Instrument Handbook, version 6.0 (Baltimore: STScI)

Rafikov, R. R., \& Draine, B. T. 2001, ApJ, 547, 207

Sommerfeld, A. 1954, Optics (New York Academic Press Inc.)

Sterken, C., \& Jaschek, C. 1996, Light Curves of Variable Stars, a pictorial Atlas (Cambridge University Press)

Tatarskii, V. I., \& Zavorotnyi, V. U. 1980, Progress in optics 18, 207

Wyithe, J. S. B., Webster, R. L., Turner, E. L., \& Mortlock, D. J. 2000, MNRAS, 315, 62 